\title{
Unitarity and causality constraints in composite Higgs models
}

\author{
Domènec Espriu and Federico Mescia \\ Departament d'Estructura i Constituents de la Matèria, Institut de Ciències del Cosmos (ICCUB), \\ Universitat de Barcelona, Martí Franquès 1, 08028 Barcelona, Spain
}

(Received 2 April 2014; published 29 July 2014)

\begin{abstract}
We study the scattering of longitudinally polarized $W$ bosons in extensions of the Standard Model where anomalous Higgs couplings to gauge sector and higher-order $\mathcal{O}\left(p^{4}\right)$ operators are considered. These new couplings with respect to the Standard Model should be thought of as the low-energy remnants of some new dynamics involving the electroweak symmetry breaking sector. By imposing unitarity and causality constraints on the $W W$ scattering amplitudes, we find relevant restrictions on the possible values of the new couplings and the presence of new dynamical resonances above $300 \mathrm{GeV}$. We investigate the properties of these new resonances and their experimental detectability. Custodial symmetry is assumed to be exact throughout, and the calculation avoids using the equivalence theorem as much as possible.
\end{abstract}

DOI: 10.1103/PhysRevD.90.015035

PACS numbers: 12.60.-i, 12.60.Fr, 12.39.Fe

\section{INTRODUCTION}

Providing tools to assess the nature of the Higgs-like boson discovered at the LHC [1,2] is probably the most urgent task that theorists face in our time. New runs will in due time clarify whether the Higgs particle is truly elementary or there is a new scale of compositeness associated to it. In the latter case, there should be a new strongly interacting sector, an extension of the Standard Model (SM) that conventionally is termed the extended electroweak symmetry breaking sector (EWSBS). All evidence suggests that the scale possibly associated to the EWSBS may be substantially larger than the electroweak scale $v=246 \mathrm{GeV}$, but it should not go beyond a few TeV. Otherwise the mass of its lightest scalar resonance becomes unnatural and very difficult to sustain [3].

Of course the Higgs could be elementary and the minimal Standard Model (MSM) realized in nature, but then some fundamental questions of elementary particle physics would remain unanswered; there would be no natural dark matter candidate-not even an axion-no hope of understanding the flavor puzzle, and perhaps even the vacuum of the theory would be unstable and jeopardize our whole picture of the Universe (see Ref. [4] for updated results).

Effective Lagrangians of Higgs and gauge bosons have already been extensively used to study current LHC data $[5,6]$ combining also in some case large electron positron collider (LEP) and flavor data. This approach has the advantage to be model independent, but the drawback is that the number of operators is usually large, and the choice of a convenient basis is the subject of intense debate [7]. Here, we are only interested in $W W$ scattering and work in the custodial limit. Therefore, only a restricted number of operators have to be considered. The effective Lagrangian is

$$
\begin{aligned}
\mathcal{L}= & -\frac{1}{2} \operatorname{Tr} W_{\mu \nu} W^{\mu \nu}-\frac{1}{4} \operatorname{Tr} B_{\mu \nu} B^{\mu \nu}+\frac{1}{2} \partial_{\mu} h \partial^{\mu} h-\frac{M_{H}^{2}}{2} h^{2}-d_{3}(\lambda v) h^{3}-d_{4} \frac{\lambda}{4} h^{4} \\
& +\frac{v^{2}}{4}\left(1+2 a\left(\frac{h}{v}\right)+b\left(\frac{h}{v}\right)^{2}+\cdots\right) \operatorname{Tr} D_{\mu} U^{\dagger} D^{\mu} U+\sum_{i=0}^{13} a_{i} \mathcal{O}_{i},
\end{aligned}
$$

where

$$
U=\exp \left(i \frac{w \cdot \tau}{v}\right) \quad \text { and }, \quad D_{\mu} U=\partial_{\mu} U+\frac{1}{2} i g W_{\mu}^{i} \tau^{i} U-\frac{1}{2} i g^{\prime} B_{\mu}^{i} U \tau^{3}
$$

Here, the $w$ are the three Goldstones of the global group $S U(2)_{L} \times S U(2)_{R} \rightarrow S U(2)_{V}$. This symmetry breaking is the minimal pattern to provide the longitudinal components to the $W^{ \pm}$and $Z$ and emerging from phenomenology. Here, the Higgs field $h$ is a gauge and $S U(2)_{L} \times S U(2)_{R}$ singlet. Larger symmetry group could be adopted [8], and consequently further Goldstone bosons may exist-the Higgs might be one of them. But all of them eventually should acquire masses, drop from an extended unitary matrix $U$, and could be parametrized by a polynomial expansion. The operators $\mathcal{O}_{i}$ include the complete set of $\mathcal{O}\left(p^{4}\right)$ operators defined in Refs. $[9,10]$. Of these only two, 
$O_{4}$ and $O_{5}$, will contribute to $W_{L} W_{L}$ scattering in the custodial limit,

$$
\mathcal{O}_{4}=\operatorname{Tr}\left[V_{\mu} V_{\nu}\right] \operatorname{Tr}\left[V^{\mu} V^{\nu}\right] \quad \mathcal{O}_{5}=\operatorname{Tr}\left[V_{\mu} V^{\mu}\right] \operatorname{Tr}\left[V_{\nu} V^{\nu}\right],
$$

where $V_{\mu}=\left(D_{\mu} U\right) U^{\dagger}$. When writing Eq. (1), we assumed the well-established chiral counting rules to limit the number of operators to the $\mathcal{O}\left(p^{4}\right)$ ones.

The parameters $a$ and $b$ control the coupling of the Higgs to the gauge sector [8]. Couplings containing higher powers of $h / v$ do not enter $W W$ scattering, and they have not been included in Eq. (1). We have also introduced two additional parameters, $d_{3}$, and $d_{4}$, that parametrize the three- and four-point interactions of the Higgs field. ${ }^{1}$ The MSM case corresponds to setting $a=b=d_{3}=d_{4}=1$ in Eq. (1). Current LHC results give the following bounds for $a, a_{4,5}$,

$$
\begin{aligned}
a & =[0.67,1.33], \quad a_{4}=[-0.094,0.10], \\
a_{5} & =[-0.23,0.26] \quad 90 \% \text { C.L.; }
\end{aligned}
$$

see Refs. $[6,12] .^{2}$ The present data clearly favor values of $a$ close to the MSM value, while the $a_{4}$ and $a_{5}$ are still largely unbounded. The parameter $b$ is almost totally undetermined at present. Other very important parameters are $a_{1}, a_{2}$, and $a_{3}$, entering the oblique and triple gauge coupling. Bounds on the oblique corrections are quite constraining [13], while the triple electroweak gauge coupling has already been measured with a level of precision [14] similar to LEP. Some results on the $\gamma \gamma W^{+} W^{-}$coupling are also available [15].

When $a$ and $b$ depart from their MSM values $a=b=1$, the theory becomes unrenormalizable in the conventional sense, although at the one-loop level $W_{L} W_{L}$ scattering can be rendered finite by a suitable redefinition of the coefficients $a_{4}$ and $a_{5}$ and $a$ (together with $v, H_{H}$, and $\lambda$ ). The relevant counterterms were worked out in Refs. $[11,16]$ using the equivalence theorem $[17,18]$ (i.e., replacing longitudinally polarized $W_{L}$ and $Z_{L}$ by the corresponding Goldstone bosons $w$ and $z$ ). This approximation is appropriate to obtain the relevant counterterms for $W_{L} W_{L}$ scattering, and in Ref. [19] the renormalization is being extended to the remaining $a_{i}$ counterterms $(i \neq 4,5)$.

In this work we extend the previous analysis [10] of unitarized $W_{L} W_{L}$ scattering to the cases $a \neq 1$ and $b \neq 1$; namely, anomalous Higgs couplings to the gauge sector are

\footnotetext{
${ }^{1}$ We bear in mind that this is not the most general form of the Higgs potential, and in fact additional counterterms are needed beyond the Standard Model [11], but this does not affect the subsequent discussion for $W_{L} W_{L}$ scattering.

${ }^{2}$ Our $a$ and $a_{4,5}$ coefficients stand for $a=1-\xi c_{H} / 2$, $a_{4}=\xi^{2} c_{11}$, and $a_{5}=\xi c_{6}$ of Ref. [6]. The $c_{H}$ range comes from the values of set $\mathrm{A}$ in Table 4 , and $c_{6,11}$ are from Table 8 of Ref. [6].
}

now considered. More specifically, we will vary $a, b$ as well as the $a_{4,5}$ parameters within the experimental bounds of Eq. (4). We use the inverse amplitude method (IAM) [20] to enforce the unitarity of longitudinally polarized $W W$ amplitudes up to the $\mathcal{O}\left(p^{4}\right)$. The calculation of the amplitude is done avoiding the use of the equivalence theorem as much as possible. The reason for this is that at the relatively low energies we are considering the replacement of the $W_{L}$ and $Z_{L}$ by $w$ and $z$ is problematic in order to make accurate predictions. In the next sections, we will give examples of how misleading the equivalence theorem (ET) can be if the right kinematical conditions are not met.

As in the previous work [10], we found that new dynamical resonances can appear in the parameter space of $a_{4,5}$ for given values of $a$ and $b$, even though for values of $a>1$ the allowed region is drastically reduced by the causality constraint. More specifically, for $a \leq 1$ and $b$ free, the overall picture is very similar to the one in Ref. [10] where the case $a=b=1$ (experimentally favored so far) was studied. In the scalar channel, for example, new dynamical resonances go from masses as low as $300 \mathrm{GeV}$ to nearly as high as the cutoff of the method of $4 \pi v \simeq 3 \mathrm{TeV}$, with rather narrow widths typically from 10 to $100 \mathrm{GeV}$. In the vector channel, the lowest achievable masses range from about $600 \mathrm{GeV}$ up to the cutoff, with widths going from 5 to about $50 \mathrm{GeV}$. For $a>1$ the picture is drastically different with respect to the one in Ref. [10], since, for a large portion of the $a_{4,5}$ parameter space, many resonances have negative widths breaking causality.

It is usually expected that a new strongly interacting sector would lead to dynamical resonances in different channels, but what turned out to be a bit of a surprise in our previous work [10] and in the present for $a<1$ is that these resonances are typically narrow and very hard to detect. This appears to be directly related to the unitarization of the $W_{L} W_{L}$ scattering in the presence of the light Higgs. Searching for these dynamical resonances at LHC will be, however, very important because if none of them reveals itself below $\sim 3 \mathrm{TeV}$ virtually all $a_{4,5}$ parameter space of the anomalous couplings could be excluded. This can be an indirect way of assessing these quartic electroweak boson couplings. Actually, no direct information on $a_{4}$ and $a_{5}$ exists at present from direct measurements of the quartic electroweak boson couplings.

Unfortunately the actual signal strength of the new resonances predicted is such that they are not currently being probed in LHC Higgs search data, and consequently no relevant bounds on $a_{4}$ and $a_{5}$ can be derived at present from the existing data - a situation that may change soon. The previous considerations emphasize the importance of indirect measures of the couplings $a_{4}$ and $a_{5}$ by searching for the additional resonances coming out from our study of $W L W L$ scattering. Measuring these anomalous couplings will be one of the main tasks of the LHC run starting in 2015 . 


\section{ISOSPIN AND PARTIAL WAVE AMPLITUDES}

Here, we introduce the basic definition of our observables. We shall consistently assume our treatment that custodial symmetry is exactly preserved. This implies taking $g^{\prime}=0$ and ignoring all the $\mathcal{O}_{i}$ operators that can contribute to $W W$ scattering but $\mathcal{O}_{4}$ and $\mathcal{O}_{5}$. This approximation also allows for a neat usage of the isospin formalism and for the convergence of the partial wave amplitudes. We also disregard operators that contain matter fields as they are totally irrelevant for the present discussion.

As emphasized in Ref. [10] when dealing with longitudinally polarized amplitudes, as opposed to using the ET approximation, caution must be exercised to account for an ambiguity introduced by the longitudinal polarization vectors that do not transform under Lorentz transformations as 4-vectors. Expressions involving the polarization vector $\epsilon_{L}^{\mu}$ can not be cast in terms of the Mandlestam variables $s, t$, and $u$ until an explicit reference frame has been chosen, as they cannot themselves be written solely in terms of covariant quantities. Obviously amplitudes still satisfy crossing symmetries when they remain expressed in terms of the external 4-momenta. A short discussion on this point is placed in Appendix B.

A general amplitude, $A\left(W^{a}\left(p^{a}\right)+W^{b}\left(p^{b}\right) \rightarrow W^{c}\left(p^{c}\right)+\right.$ $\left.W^{d}\left(p^{d}\right)\right)$, can be written using isospin and Bose symmetries as

$$
\begin{aligned}
A^{a b c d} & \left(p^{a}, p^{b}, p^{c}, p^{d}\right) \\
= & \delta^{a b} \delta^{c d} A\left(p^{a}, p^{b}, p^{c}, p^{d}\right)+\delta^{a c} \delta^{b d} A\left(p^{a},-p^{c},-p^{b}, p^{d}\right) \\
& +\delta^{a d} \delta^{b c} A\left(p^{a},-p^{d}, p^{c},-p^{b}\right),
\end{aligned}
$$

with

$$
\begin{aligned}
A^{+-00} & =A\left(p^{a}, p^{b}, p^{c}, p^{d}\right) \\
A^{+-+-} & =A\left(p^{a}, p^{b}, p^{c}, p^{d}\right)+A\left(p^{a},-p^{c},-p^{b}, p^{d}\right) \\
A^{++++} & =A\left(p^{a},-p^{c},-p^{b}, p^{d}\right)+A\left(p^{a},-p^{d}, p^{c},-p^{b}\right) .
\end{aligned}
$$

The fixed-isospin amplitudes are given by

$$
\begin{aligned}
& T_{0}(s, t, u)=\langle 00|S| 00\rangle=3 A^{+-00}+A^{++++} \\
& T_{1}(s, t, u)=\langle 10|S| 10\rangle=2 A^{+-+-}-2 A^{+-00}-A^{++++} \\
& T_{2}(s, t, u)=\langle 20|S| 20\rangle=A^{++++} .
\end{aligned}
$$

We shall also need the amplitude for the process $W^{+} W^{-} \rightarrow h h$. Taking into account that the final state is an isospin singlet and defining

$$
A^{+-}=A\left(W^{+}\left(p^{+}\right)+W^{-}\left(p^{-}\right) \rightarrow h\left(p^{c}\right)+h\left(p^{d}\right)\right),
$$

the projection of this amplitude to the $I=0$ channel gives
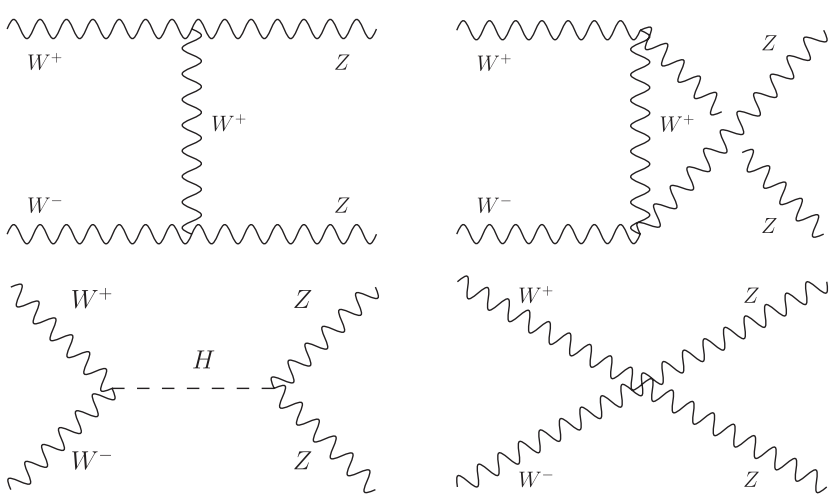

FIG. 1. Diagrams contributing to $A(s, t, u)$ at tree level.

$$
T_{H, 0}(s, t, u)=\sqrt{3} A^{+-} .
$$

The partial wave amplitudes for fixed isospin $I$ and total angular momentum $J$ are

$$
t_{I J}(s)=\frac{1}{64 \pi} \int_{-1}^{1} d(\cos \theta) P_{J}(\cos \theta) T_{I}(s, t, u),
$$

where the $P_{J}(x)$ are the Legendre polynomials and $t=(1-\cos \theta)\left(4 M^{2}-s\right) / 2, u=(1+\cos \theta)\left(4 M^{2}-s\right) / 2$ with $M$ being the $W$ mass. We will concern ourselves with only the lowest nonzero partial wave amplitude in each isospin channel: $t_{00}(s), t_{11}(s)$, and $t_{20}(s)$, namely, the scalar/isoscalar, vector/isovector, and isotensor amplitudes, respectively. Unitarity directly implies that $\left|t_{I J}(s)\right|<1$. For further implications of unitarity on $t_{I J}(s)$, the interested reader may see Ref. [21].

In this work, the partial wave amplitudes $t_{I J}(s)$ are studied up to $\mathcal{O}\left(p^{4}\right)$, namely,

$$
t_{I J}(s)=t_{I J}^{(0)}(s)+t_{I J}^{(2)}(s) .
$$

Here, $t_{I J}^{(0),(2)}(s)$ are tree level and $\mathcal{O}\left(p^{4}\right)$ contributions, respectively. $t_{I J}^{(0)}(s)$ can be constructed from Eq. (10) by using the crossing and isospin relation for the tree-level contributions of $A^{+-00}$ (Fig. 1). The analytic results of $A^{+-00}$ at tree level are in Appendix A. $t_{I J}^{(0)}(s)$ contains the anomalous coupling $a$, but $b$ does not enter at tree level. $t_{I J}^{(4)}(s)$ includes tree-level contributions from $a_{i}$ counterterms (see Appendix A for analytic result) and the one-loop corrections to the diagrams in Fig. 1. At one-loop level, the $b$ parameters enter $t_{I J}^{(4)}(s)$ by the one-loop expression of $A^{+-00}$ calculated in Ref. [16].

\section{SCRUTINY OF THE TREE-LEVEL AMPLITUDES $t_{\mathbf{0 0}}^{(\mathbf{0})}, t_{\mathbf{2 0}}^{(\mathbf{0})}$, AND $t_{\mathbf{1 1}}^{(\mathbf{0})}$}

For values of $a$ different from 1 , the $W_{L} W_{L}$ scattering amplitudes exhibit rather different behavior with respect to 

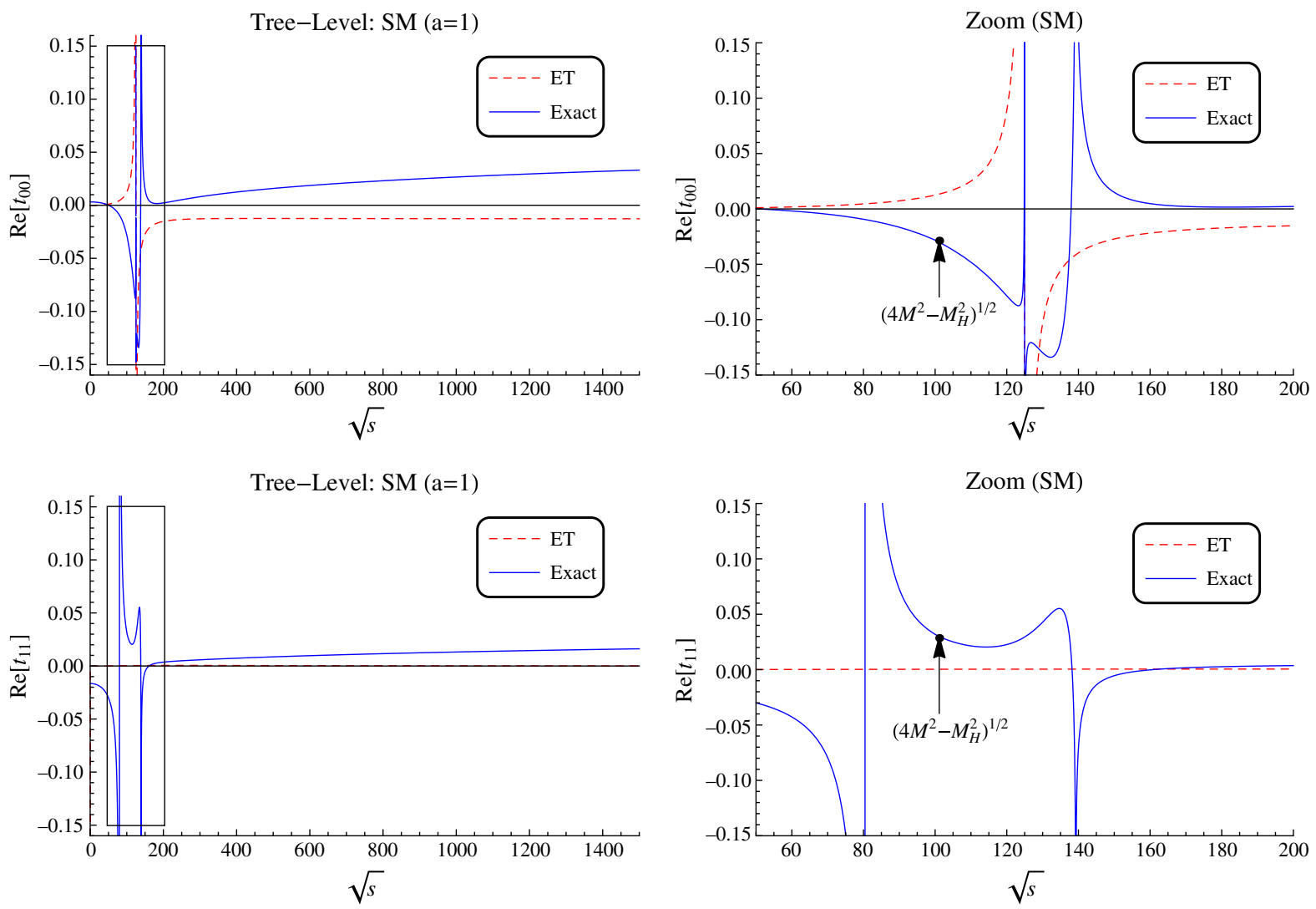

FIG. 2 (color online). Plot of $t_{00}^{(0)}$ (above) and $t_{11}$ (below) for $a=1$. In both cases a zoom on the lowest values of $s$ to show the complete analytic structure is presented. The arrow indicates the position of one of the subthreshold singularities that is invisible at the scale of the plot.

the MSM case $a=1$. The most important difference is that the $\left|t_{I J}\right|<1$ unitarity bound is violated at tree level pretty quickly. We shall see later how to restore unitarity with the help of higher loops and counterterms, but in this section we concentrate on the peculiarities of the tree-level amplitudes $t_{00}^{(0)}, t_{20}^{(0)}$, and $t_{11}^{(0)}$. Here, the partial wave amplitudes are studied in the complete theory, namely, away from the ET approximation. This is a key point since there are interesting kinematical features of $t_{I J}^{(0)}$ that are totally missed in the ET approximation, such as the presence of subthreshold singularities and zeroes of $t_{I J}^{(0)}$ absent in the ET approximation. Some of these features will be crucial in our analysis.

To study the behavior of $t_{I J}^{(0)}$, we will establish three different regions according to the range of the values of $a=1, a>1$, and $a<1$.

\section{A. Case $a=1$}

In Fig. 2 we plot the tree-level isoscalar partial wave amplitude $t_{00}^{(0)}(s)$ for $W_{L} W_{L} \rightarrow Z_{L} Z_{L}$ as a function of $s$. The external $W$ legs are taken on shell $\left(p^{2}=M^{2}=M_{W}^{2}=M_{Z}^{2}\right)$. As we see from Fig. 2 the partial wave amplitude has a rather rich analytic structure. It has one pole at $s=M_{H}^{2}$, but also a second singularity can be seen at the value $s=3 M^{2}$. A closer examination reveals also a third singularity at $s=4 M^{2}-M_{H}^{2}$, invisible in Fig. 2 as it happens to be multiplied by a very small number. These singularities correspond to poles of the $t$ - and $u$ channel diagrams in Fig. 1 that, after the angular integration of Eq. (10) to obtain the partial wave amplitudes, behave as logarithmic divergences. The $t$ and $u$ channels are absent in the ET approximation. Note that both singularities are below the physical threshold at $s=4 M^{2}$. Beyond the $s=$ $3 M^{2}$ singularity, the amplitude for $a=1$ is always positive, as can be seen in Fig. 2 .

In Fig. 2 we also plot the tree-level partial wave amplitude $t_{11}^{(0)}(s)$. Here, a pole at $s=M^{2}$ is visible, as expected, along with the two kinematical subthreshold singularities already mentioned. In Fig. 2 the $t_{00}^{(0)}$ and $t_{11}^{(0)}$ amplitudes are also compared with the respective amplitudes obtained in ET approximation (computed assuming $M=0$ as is customary). As can be seen, the ET is grossly inadequate at low energies. In particular it fails in reproducing the rich analytic structure of the amplitudes. The nonanalyticity at $s=3 M^{2}$ and $s=4 M^{2}-M_{H}^{2}$ due to subthreshold singularities is actually also present in the $t_{20}^{(0)}$ partial wave amplitude (not depicted), corresponding like in the other two cases to a (zero width) logarithmic 

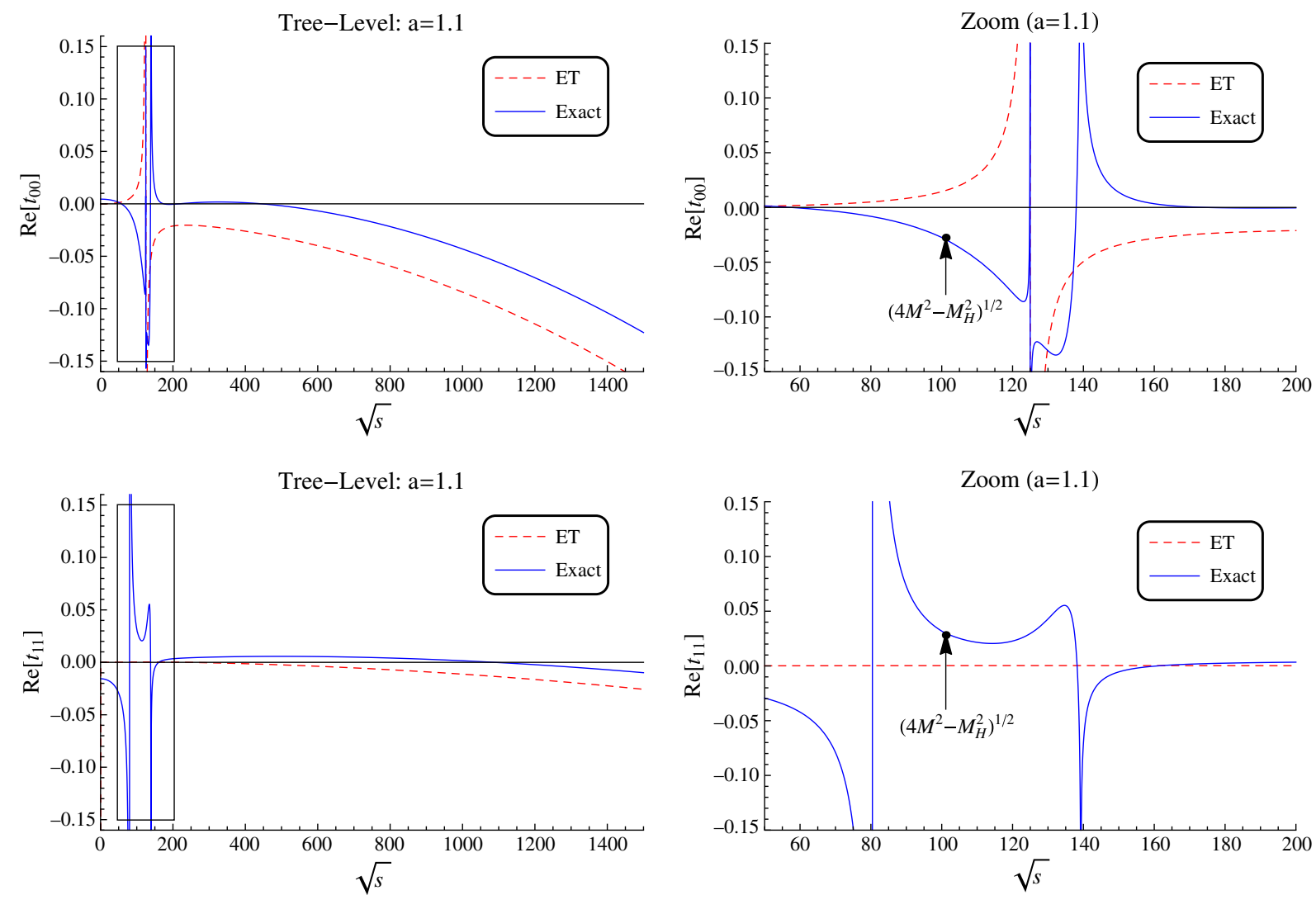

FIG. 3 (color online). Plot of $t_{00}^{(0)}$ and $t_{11}^{(0)}$ for $a=1.1$ and a zoom on the low $s$ region where the amplitude is very small. The amplitudes are not unitarity, since they diverge at large $s$. Moreover, they are vanishing above threshold, e.g., $\sqrt{s}=2 M_{W}$. The ET result is also shown by a (red) dotted line.

pole. In $t_{20}^{(0)}$ there are no other singularities as no $I=2$ particle is exchanged in the $s$ channel. These subthreshold singularities are genuine effects in the $W_{L} W_{L} \rightarrow Z Z$ amplitudes and are independent from the value of $a$. These features are conspicuously absent in the analogous amplitude computed in the ET.

$W_{L} W_{L} \rightarrow Z_{L} Z_{L}$ scattering can be accessible at the LHC by the studying the process $p p \rightarrow W W j j$. Then, these subthreshold singularities should be hardly visible mostly due to the off-shellness of the $W_{L} W_{L} \rightarrow Z_{L} Z_{L}$ amplitude on $p p \rightarrow W W j j$. The experimental process spreads the logarithmic poles over a range of invariant masses. For instance, the singularity at $s=M^{2}$ appears actually at $s=$ $\sum q_{i}^{2}-M^{2}$ if $W$ legs are off shell. In addition cuts in $p_{T}$ should render the partial wave amplitude actually nonsingular. ${ }^{3}$

\section{B. Case $a>1$}

The three subthreshold singularities appearing at $a=1$ are also present in this case. However, for $a>1$ the partial wave amplitudes also show new features. First of all, as

\footnotetext{
${ }^{3}$ We thank D. D'Enterria and X. Planells for discussions on these points.
}

shown in Fig. 3 for $a=1.1$ and amplitudes $t_{00}^{(0)}(s)$ and $t_{11}^{(0)}(s)$, the tree-level partial wave amplitude for $t_{I J}^{(0)}(s)$ shows clear nonunitary behaviors as it goes to $-\infty$ as $s$ increases. In addition, for $a>1$ the tree-level partial wave amplitudes for $t_{I J}^{(0)}(s)$ have zeroes for values of $s$ above threshold and well below the cutoff scale $(3 \mathrm{TeV})$ of our effective Lagrangian. Setting, for example, the value $a=$ 1.1 compatible with the experimental constraint in Eq. (4), the $t_{00}^{(0)}(s)$ amplitudes vanish at two values of $\sqrt{s}$ around 216 and $445 \mathrm{GeV}$ (see Fig. 3 for $a=1.1$ ), the $t_{11}^{(0)}(s)$ at a value around $1 \mathrm{TeV}$, as well as $t_{20}^{(0)}(s)$ at about $800 \mathrm{GeV}$ (not shown). For $a>1.125$ the tree-level amplitude $t_{00}^{(0)}$ has no zeroes (Fig. 4 for $a=1.3$ ), whereas the $t_{11}^{(0)}(s)$ and $t_{20}^{(0)}(s)$ amplitudes for values of $a$ compatible with bounds in Eq. (4) still vanish at specific values of $\sqrt{s}$. For example, for $a=1.3$ the zeroes of $t_{11}^{(0)}(s)$ and $t_{20}^{(0)}(s)$ are at $\sqrt{s}$ around $450 \mathrm{GeV}$. The presence of zeroes for the tree-level amplitudes at low values of $\sqrt{s}$ is an interesting point as it means that around these zeroes the $W_{L} W_{L} \rightarrow Z_{L} Z_{L}$ amplitudes are strongly suppressed. It may be relevant to note that the $t_{00}^{(0)}$ and $t_{11}^{(0)}$ amplitudes are very small over a fairly extended range of values of $s$ for a range of values of 

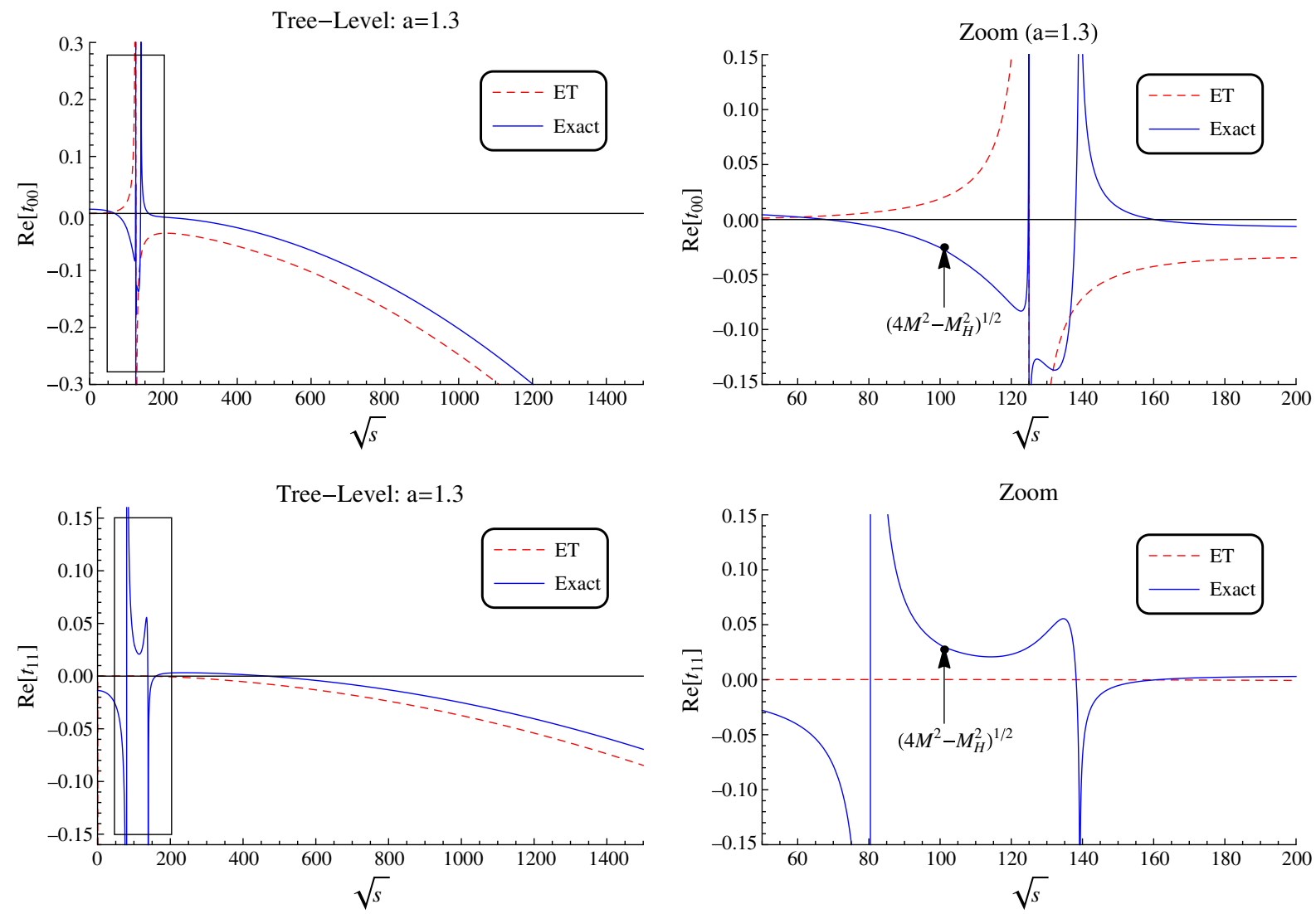

FIG. 4 (color online). Plot of $t_{00}^{(0)}$ and $t_{11}^{(0)}$ for $a=1.3$. Amplitudes are not unitarity. The three singularities commented on the text are shown in each channel. Amplitudes are not vanishing for values of $s$ up to the range of validity of the effective Lagrangian. The ET results are indicated by a dotted line.

$a>1$ (particularly so in the isovector channel). These facts could perhaps be used to set rather direct bounds on this particular coupling. This issue deserves further phenomenological study.

\section{Case $a<1$}

For $a<1$ the $t_{I J}^{(0)}$ amplitude still presents the two subthreshold singularities at $s=3 M^{2}$ and $s=4 M^{2}-$ $M_{H}^{2}$. Beyond them, however, no additional zeroes appear, and amplitudes are positive and go to $\infty$ as $s$ increases. This clearly reflects the nonunitary character of $t_{I J}^{(0)}$ amplitudes for $a \neq 1$. In Fig. 5, we show as an example the $t_{11}^{(0)}(s)$ and $t_{20}^{(0)}(s)$ amplitudes in the case $a=0.9$. The equivalent amplitudes computed by making use of the ET are also shown in Fig. 5. Both in this $a<1$ case and in the $a>1$ one, we see that the ET works reasonably well for large values of $s$, but again fails at low and moderate values.

\section{UNITARITY CORRECTIONS}

In the case of Higgs anomalous couplings to the gauge sector $(a \neq 1$ and $b \neq 1)$, the tree-level amplitudes $t_{I J}^{(0)}$ are nonunitarity, and we are forced to include additional operators in the theory, such as the $a_{i}$ counterterms in Eq. (1). At one-loop level, the $a_{i}$ will cancel the divergences of the Lagrangian in Eq. (1), and finite couplings renormalized at some UV scale will remain $[11,16]$, namely,

$$
\begin{aligned}
& \left.a_{4}\right|_{\text {finite }} \simeq \frac{1}{(4 \pi)^{2}} \frac{-1}{12}\left(1-a^{2}\right)^{2} \log \frac{v^{2}}{f^{2}} \\
\left.a_{5}\right|_{\text {finite }} \simeq & \frac{1}{(4 \pi)^{2}} \frac{-1}{24}\left[\left(1-a^{2}\right)^{2}+\frac{3}{2}\left(\left(1-a^{2}\right)-(1-b)\right)^{2}\right] \\
& \times \log \frac{v^{2}}{f^{2}}
\end{aligned}
$$

where $f$ is the scale of the new interactions, and possibly other finite pieces.

$\mathrm{Up}$ to now, the calculation of the one-loop $t_{I J}^{(2)}(s)$ contribution in Eq. (11) is not available for $a$ and $b$ arbitrary and longitudinally polarized $W$ and $Z$. This would require the evaluation of over 1000 diagrams. A numerical calculation is only available in Ref. [22] for the case $a=b=1$, but it is not very useful for our purposes.

For this reason, to estimate the $t_{I J}^{(2)}(s)$ contribution in Eq. (11), we proceed in the following way. The analytic 

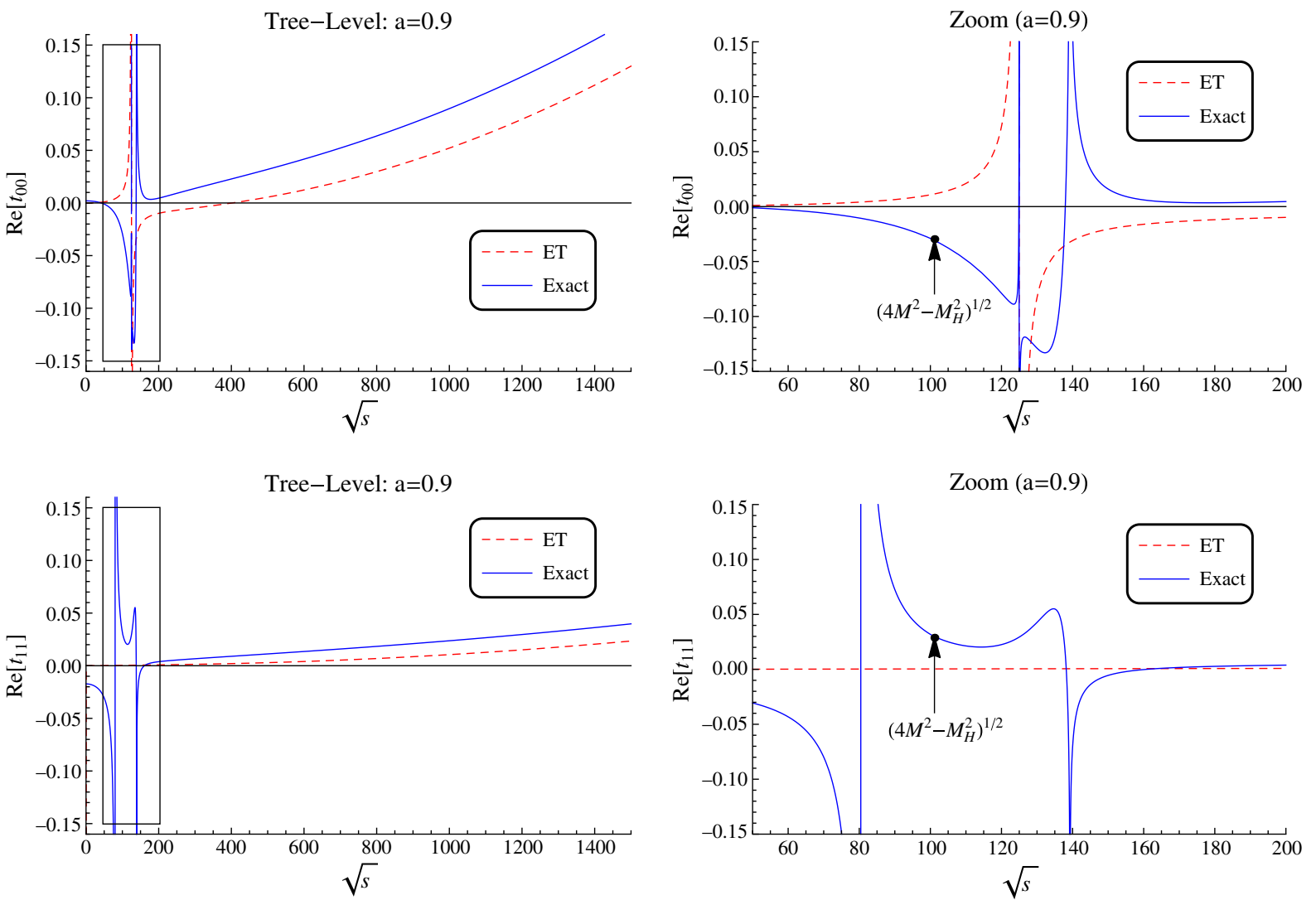

FIG. 5 (color online). Plots of $t_{00}^{(0)}$ and $t_{11}^{(0)}$ for $a=0.9$ and a zoom of the region at low $s$ where the amplitudes are very small. No additional zeroes appear, and the amplitudes also show a nonunitary behavior at large $s$. The nearly invisible logarithmic singularity at $s=4 M^{2}-M_{H}^{2}$ is indicated. The results in the ET approximation are also indicated by a dotted line.

contribution from $a_{4,5}$ terms are calculated exactly with longitudinally polarized $W$ and $Z$ (Appendix A) like the tree-level contribution $t_{I J}^{(2)}(s)$. The real part of $t_{I J}^{(2)}(s)$ will, however, be determined using the ET [17,18]; i.e., we replace this loop amplitude by the corresponding process $w^{+} w^{-} \rightarrow z z$. For this part of the calculation, we take $q^{2}=0$ for external legs and set $M=0$, but the Higgs mass is kept. The relevant diagrams of $A(w w \rightarrow z z)$ entering $t_{I J}^{(4)}(s)$ were calculated in Ref. [16] where explicit expressions for the different diagrams for arbitrary values of the couplings $a$ and $b$ can be found. This calculation was checked and extended in Ref. [11], albeit setting $M_{H}=0$. As to the imaginary part of $t_{I J}^{(2)}(s)$, we can take advantage of the optical theorem to circumvent the problem of using the ET approximation. In the $I=1, J=1$ and $I=2, J=0$ cases, we can use the relations

$$
\operatorname{Im} t_{I J}^{(2)}(s)=\sigma(s)\left|t_{I J}^{(0)}(s)\right|^{2},
$$

while for the $I=0$ amplitude, we also have a contribution from a two-Higgs intermediate state. Then

$$
\operatorname{Im} t_{00}(s)=\sigma(s)\left|t_{00}(s)\right|^{2}+\sigma_{H}(s)\left|t_{H, 0}(s)\right|^{2},
$$

with

$$
\sigma(s)=\sqrt{1-\frac{4 M^{2}}{s}}, \quad \sigma_{H}(s)=\sqrt{1-\frac{4 M_{H}^{2}}{s}} .
$$

We believe that, for the purpose of identifying dynamical resonances, normally occurring at $s \gg M_{H}^{2}$, the approximation of relying on the ET for the real part of the loops is fine. Note that the dominant contribution to the real part for large $s$, of order $s^{2}$, is controlled by the contribution coming from couplings $a_{4,5}$. We have also actually checked that, unless $a_{4}$ and $a_{5}$ are both very small, the contribution from the real part of the loop amounts only to a small correction to $t_{I J}^{(2)}$.

The final ingredient we need is a procedure to construct a unitary amplitude that perturbatively coincides with the tree plus one-loop result but incorporates the principle of unitarity. To this purpose we use the IAM [20] to the amplitude in Eq. (11), namely,

$$
t_{I J} \approx \frac{t_{I J}^{(0)}}{1-t_{I J}^{(2)} / t_{I J}^{(0)}},
$$

which is identical to the [1,1] Padé approximant to $t_{I J}$ derived from Eq. (11). The above expression obviously reproduces the first two orders of the perturbative 
expansion [Eq. (11)] and, in addition, satisfies the necessary unitarity constraints, namely, $\left|t_{I J}\right|<1$ at high energies and

$$
\operatorname{Im} t_{I J}(s)=\sigma(s)\left|t_{I J}(s)\right|^{2},
$$

when the perturbative ingredients satisfy

$$
\operatorname{Im} t_{I J}^{(2)}(s)=\sigma(s)\left|t_{I J}^{(0)}(s)\right|^{2},
$$

as they must from the optical theorem. We refer to Ref. [10] and references therein for a more detailed discussion. We also recommend reading the recent Ref. [23] for a rather complete review. In what follows we shall adhere to the procedure outlined in Ref. [10].

There is no really unambiguous way of applying the IAM to the case where there are coupled channels with different thresholds. This will be relevant to us only in the $t_{00}$ case as there is an intermediate state consisting of two Higgs particles. Here, we shall adhere to the simplest choice that consists of assuming Eq. (17) to remain valid also in this case. In addition, there is a decoupling of the two $I=0$ channels in the case $a^{2}=b$, as also discussed in Ref. [23] in the context of the ET approximation. We have checked our results for different values of $b$; in particular we see that setting $b=a^{2}$ does not give for the resonances that are eventually found results that are noticeably different from those obtained for other values of $b$. Finally, we have also checked explicitly the unitarity of our results.

\section{LOOKING FOR RESONANCES IN $a_{4}$ AND $a_{5}$ PARAMETER SPACE}

Nonrenormalizable models such as the effective theory described by the Lagrangian (1) typically produce scattering amplitudes that grow too fast with the scattering energy breaking the unitarity bounds [21] at some point or other.

Chiral descriptions of QCD [24] are archetypal examples of this behavior, and unitarization techniques have to be used to recover unitarity. The IAM [20], described in the previous section, is a convenient way of doing so. In QCD when the physical value of the pion decay constant $f_{\pi}$ and the $\mathcal{O}\left(p^{4}\right)$ low-energy terms $L_{i}$ (as defined, e.g., in Ref. [24], the counterpart of the $a_{i}$ in strong interactions) are inserted in the chiral Lagrangian and the IAM method is used, the validity of the chiral expansion is considerably extended, and one is able to reproduce the $\rho$ meson pole as well as many other properties of low-energy QCD [20]. The limitations of the method derive to a large extent from the accuracy in our knowledge of the different amplitudes entering the game. Different unitarization methods (such as, e.g., N/D expansions or the Roy equations) always give very similar results as far as the first dynamical resonances are concerned.

Any strongly interacting theory should exhibit an infinite number of resonances. This is what hopefully one would get if all the terms in the effective expansion were included, including all loop corrections and counterterms. Including contributions up to $\mathcal{O}\left(p^{4}\right)$, our expression of $t_{I J}(s)$ is to a large extent polynomials up to order $s^{2}$ (module logs). Therefore, we can find one or two resonances- the lowestlying ones in each channel. However, this is already providing us precious information on the dynamics of the strongly interacting theory. In the present case, the mere presence of higher dynamical resonances signals gives interesting information on the higher-order coefficients of the effective Lagrangian (1) and therefore on $W W$ scattering.

If instead of a new strongly interacting sector the EWSBS is perturbative, with pointlike fields (a possibility could be an extended scalar sector or two Higgs-doublet models), integrating them out would yield nonvanishing values for the coefficients $a_{4}$ and $a_{5}$ [25]. The unitarization method then reproduces approximately the masses of the particles that were originally integrated out, which is still valid information for physics beyond the SM.

To find resonances we perform a scan for the values $\left|a_{4}\right|<0.01$ and $\left|a_{5}\right|<0.01$ and $a$ and $b$ fixed looking for the presence or otherwise of resonances in the different channels. We will consider the different cases for $a \neq 1$ since the case $a=1$ was discussed in detail in Ref. [10].

When looking for dynamical resonances, we use two different methods. First we look for a zero of the real part of the denominator in Eq. (17) and use the optical theorem to determine the imaginary part—i.e., the width—at that location. A second method consists of searching directly for a pole in the complex plane. In our case both methods give very similar results, the reason being that the widths are typically quite small. It should be stated right away that, because of the way we compute the full amplitude, with separate derivations of the real and the imaginary parts, the analytic continuation to the whole complex plane for $s$ is somewhat ambiguous. Had we found large imaginary parts, some doubts could be cast on the results, but fortunately this is not the case in virtually all of parameter space. Of course, a mathematical zero in the denominator (i.e., a genuine pole in the amplitude $t_{I J}$ ) is sometimes very difficult to get numerically, but proper resonances tend to reveal themselves in a rather clear way, nevertheless. Some difficult cases present themselves for $a>1$ when the putative resonance is close to one of the zeroes of the treelevel amplitude that appears in this case, and we had to study these situations carefully.

Physical resonances must have a positive width and are only accepted as genuine resonances if $\Gamma<M / 4$. Theories with resonances having a negative width violate causality, and the corresponding values of the low-energy constants in the effective theory are to be rejected as leading to unphysical theories. No meaningful microscopic theory could possibly lead to these values for the effective couplings. 


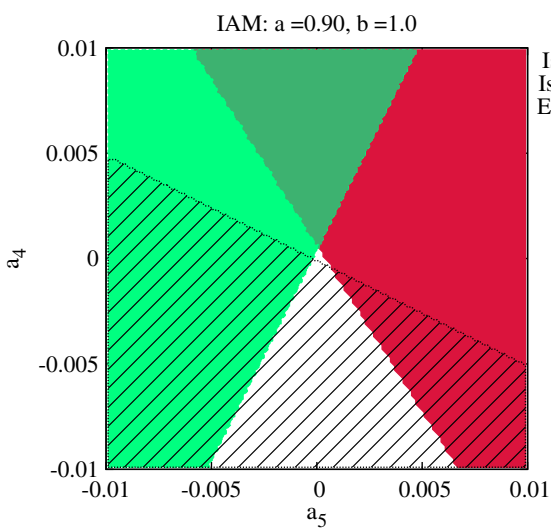

(a)

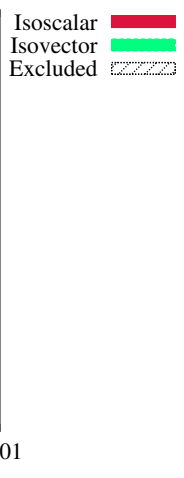

Excluded

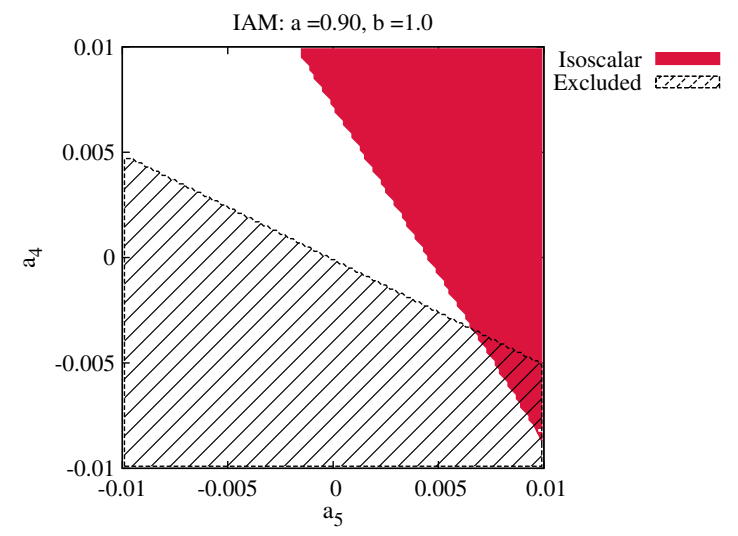

(b)

FIG. 6 (color online). For $a=0.9$ and $b=1$ : (a) Regions with isoscalar and isovector resonances (and the isotensor exclusion region) up to a scale $4 \pi v \approx 3 \mathrm{TeV}$. (b) Same as (a), but only showing isoscalar/isovector resonances in which $M_{S, V}<600 \mathrm{GeV}$, for comparison with current Higgs search results.

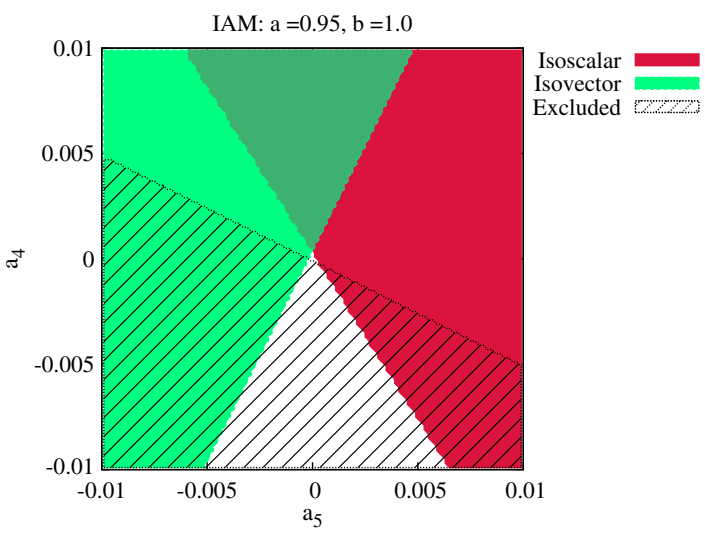

(a)

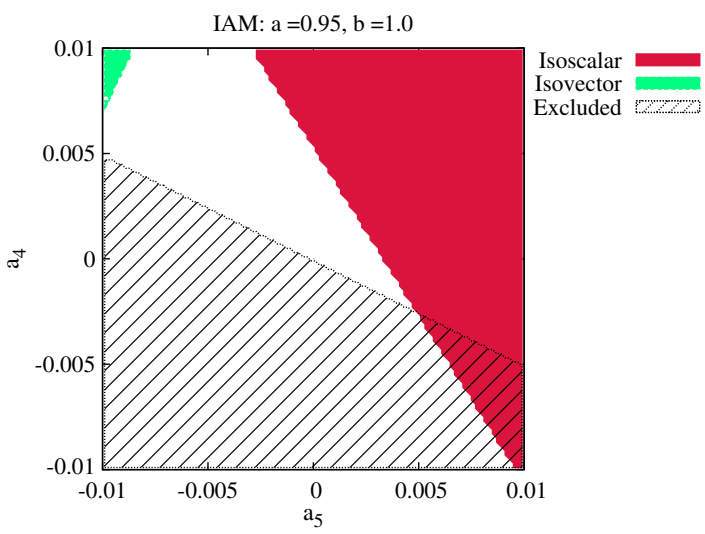

(b)

FIG. 7 (color online). For $a=0.95$ and $b=1$ : (a) Regions with isoscalar and isovector resonances (and the isotensor exclusion region) up to a scale $4 \pi v \approx 3 \mathrm{TeV}$. (b) Same as (a), but only showing isoscalar/isovector resonances in which $M_{S, V}<600 \mathrm{GeV}$, for comparison with current Higgs search results.

\section{A. Case $a<1$}

We start by considering this case where the unitarized amplitudes $t_{00}^{(0)}, t_{11}^{(0)}$, and $t_{20}^{(0)}$ share some properties with the ones from Ref. [10] for $a=1$; namely, the tree-level amplitude has no zeros beyond the kinematical singularity existing at $s=3 M^{2}$. In this case the sign of the tree-level amplitude as $s \rightarrow \infty$ is always positive in our conventions.

First of all we look for the existence of resonances. We set $b=1$ and consider two values ${ }^{4} a=0.9$ and $a=0.95$ compatible with experimental bounds, and indeed we easily find resonances in various channels. Most of them have the right causality properties that make the theory acceptable. However, in the $I=2, J=0$ channel, we see that there is a region in the $a_{4}-a_{5}$ plane where causality is violated. This

\footnotetext{
${ }^{4}$ Other values of $a$ have also been studied, but we here present results only for these two.
}

corresponds to the shaded region in the lower part of Figs. 6, 7, and 8, and the theories corresponding to these values for the parameters $a_{4}, a_{5}$ are therefore not acceptable. The presence of this excluded region is in exact correspondence with what was found for the $a=1$ case in Ref. [10] (and also with a similar situation in pion physics [20]).

In Fig. 6 we show the region of parameter space in $a_{4}$, $a_{5}$ where isoscalar and isovector resonances exist for the value $a=0.9$ along with the isotensor exclusion region. The pattern here has some analogies with the case $a=1$ studied in Ref. [10], but proper ${ }^{5}$ resonances are somewhat harder to form; in particular, in the vector channel no resonance is found below $600 \mathrm{GeV}$ for $a=0.9$ in contrast to the $a=1$ case studied in Ref. [10]. For $a=0.95$ some

\footnotetext{
${ }^{5}$ Recall that resonances are required to have, in addition to the correct causal properties, $\Gamma<M / 4$.
} 


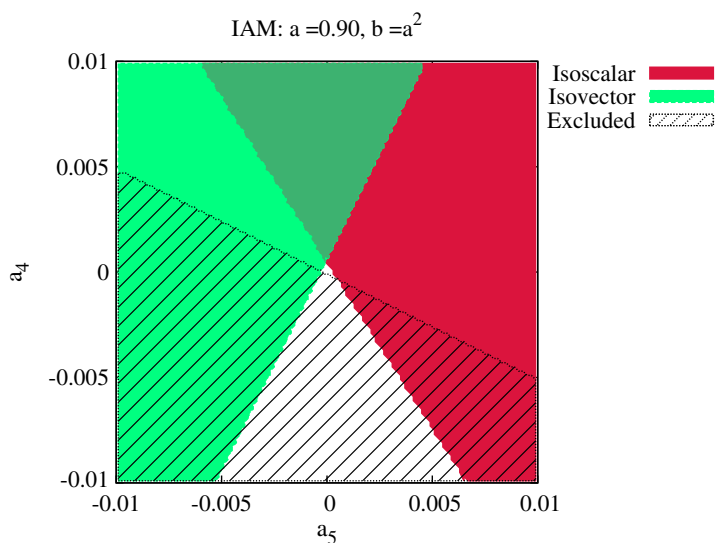

(a)

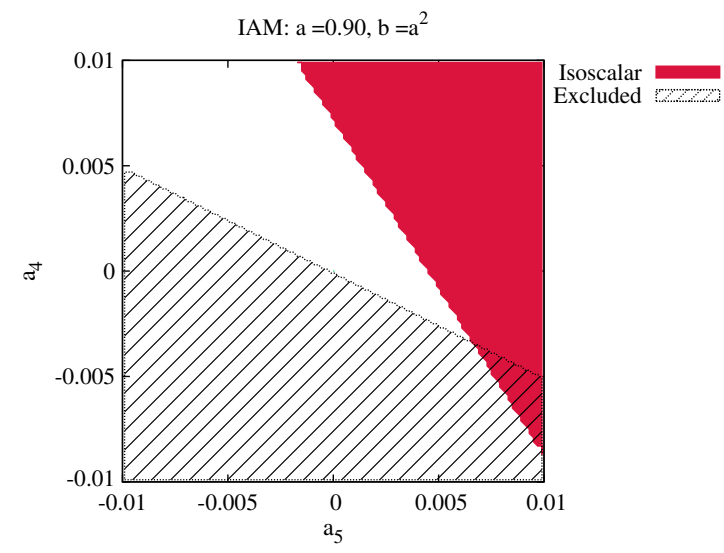

(b)

FIG. 8 (color online). For $a=0.9$ and $b=a^{2}$ : (a) Regions with isoscalar and isovector resonances (and the isotensor exclusion region) up to a scale $4 \pi v \approx 3 \mathrm{TeV}$. (b) Same as (a), but only showing isoscalar/isovector resonances in which $M_{S, V}<600 \mathrm{GeV}$. This can be compared with Fig. 6 to conclude that $b$ has very little relevance here.

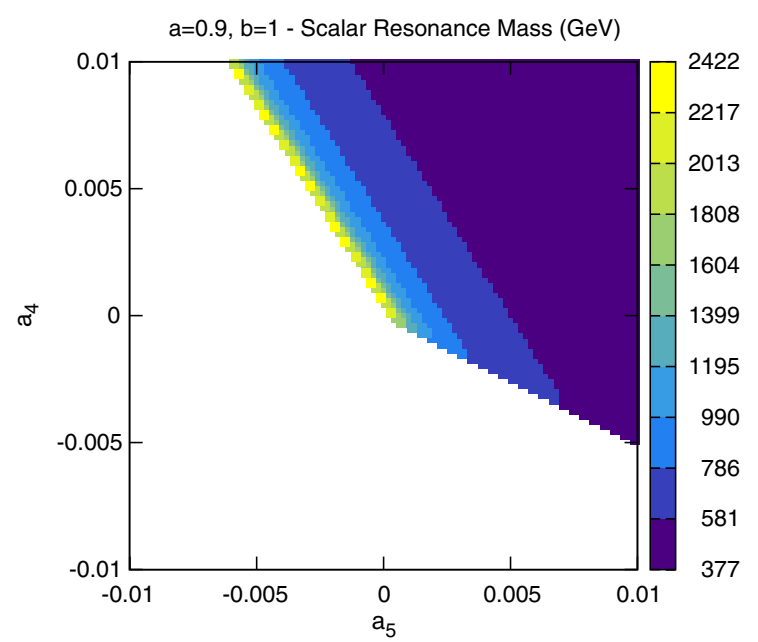

(a)

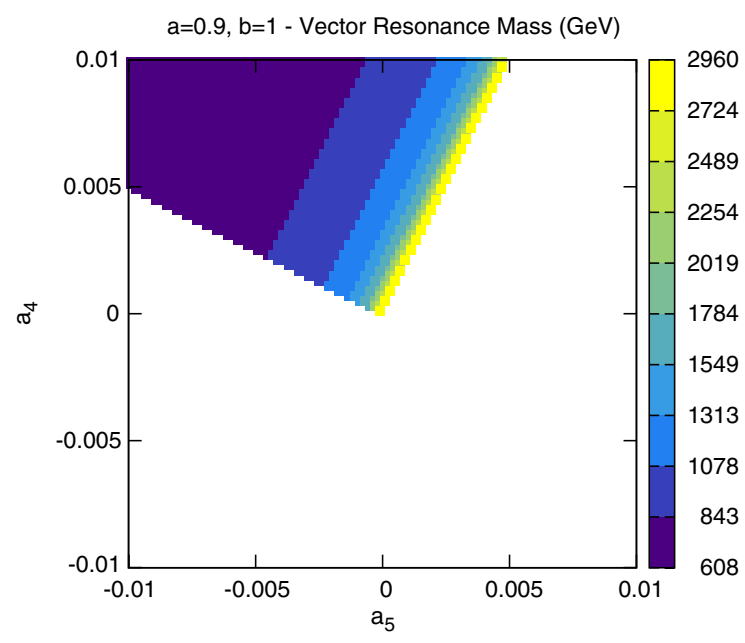

(c)

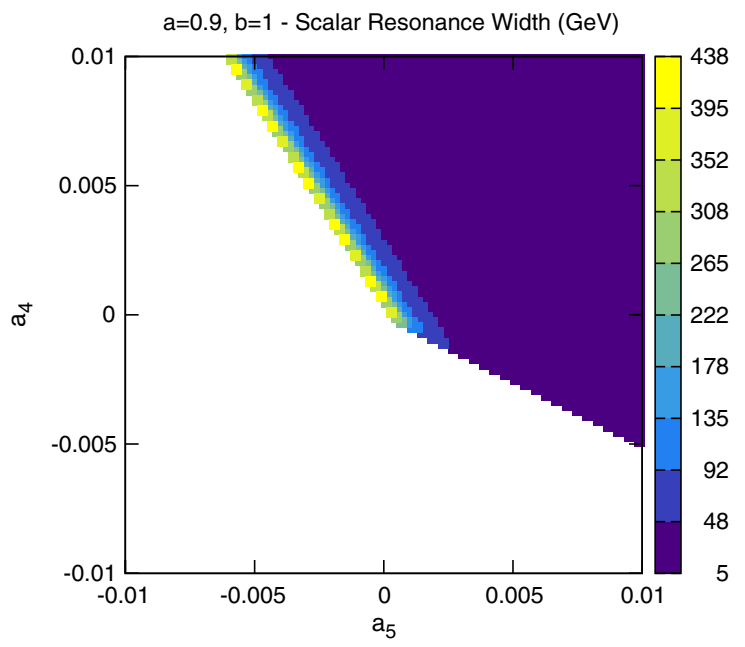

(b)

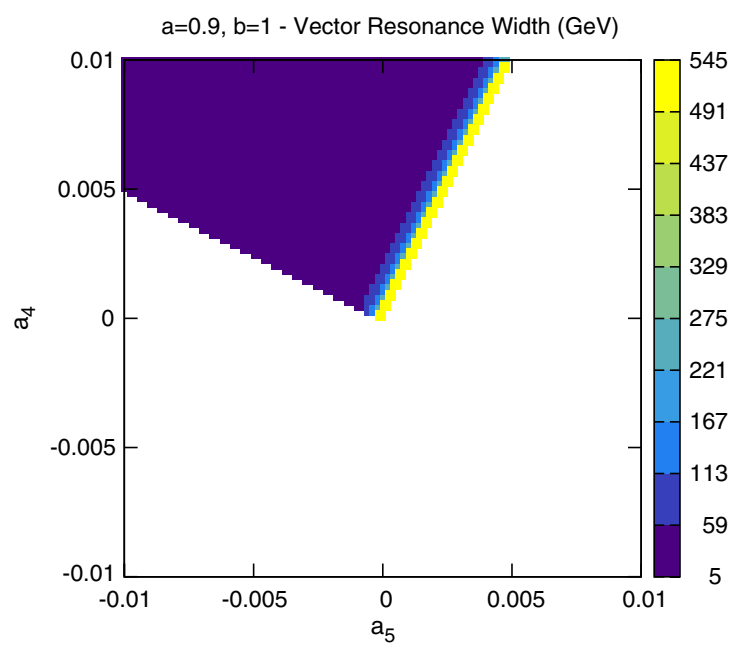

(d)

FIG. 9 (color online). For $a=0.9$ and $b=1$, masses in GeV for (a) scalar and (b) vector resonances predicted from the unitarized partial wave amplitudes of $W W \rightarrow W W$ scattering. Widths in $\mathrm{GeV}$ for the corresponding (c) scalar and (d) vector resonances. 


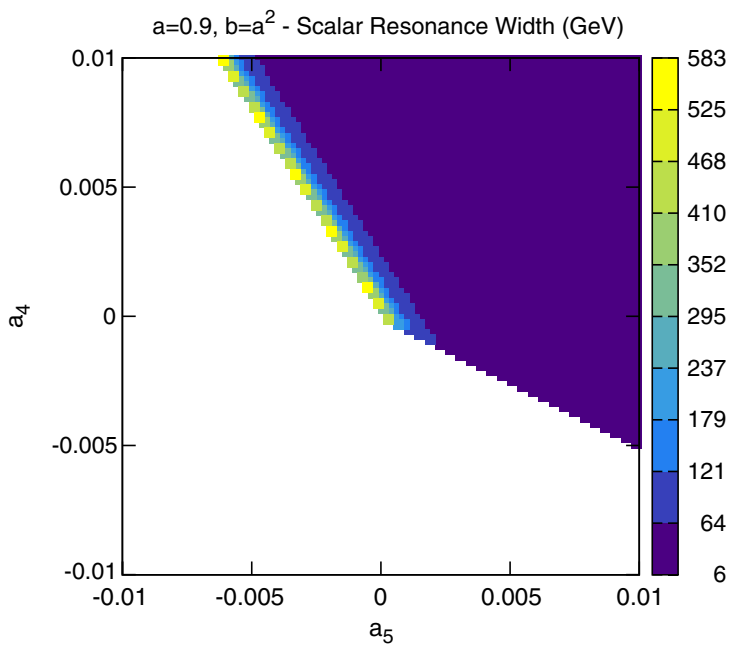

(a)

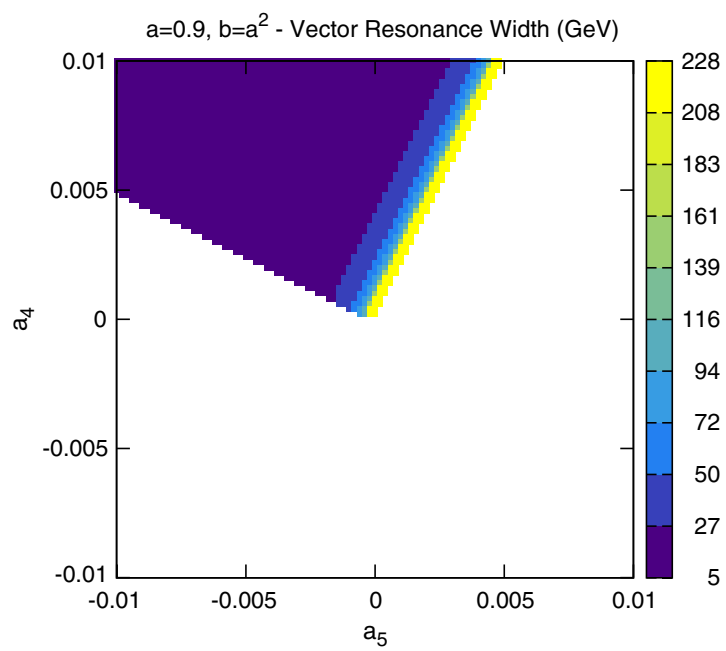

(b)

FIG. 10 (color online). For $a=0.9$ and $b=a^{2}$ : Widths of the (a) isoscalar and (b) isovector resonances. Comparison with the equivalent plots of the previous figure shows some influence of $b$ here.

vector resonances can be found below $600 \mathrm{GeV}$ for rather extreme values of $a_{4}$ and $a_{5}$ (upper left corner in Fig. 7)

If no resonances are found at the LHC all the way up to $3 \mathrm{TeV}$, the values of $a_{4}$ and $a_{5}$ in the colored regions in

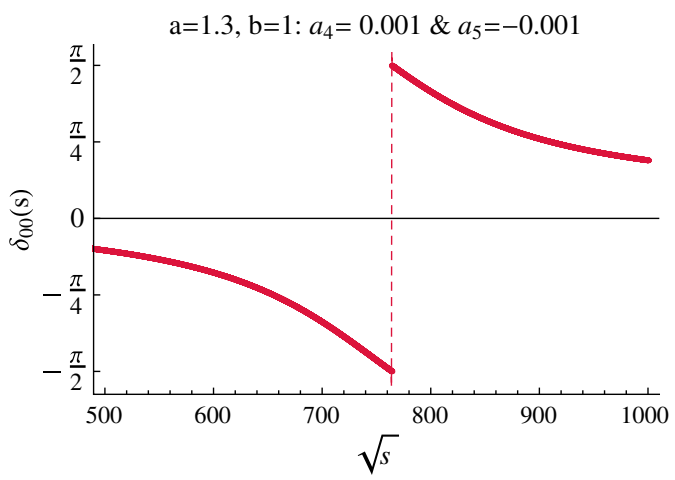

(a) the left figures of Figs. 6 and 7 could be excluded, and then $a_{4}$ and $a_{5}$ should lie within the small central region in the left plots of Figs. 6 and 7. As small as these regions are, they are noticeably larger than the one corresponding to $a=1$, which was virtually nonexistent. This is true even

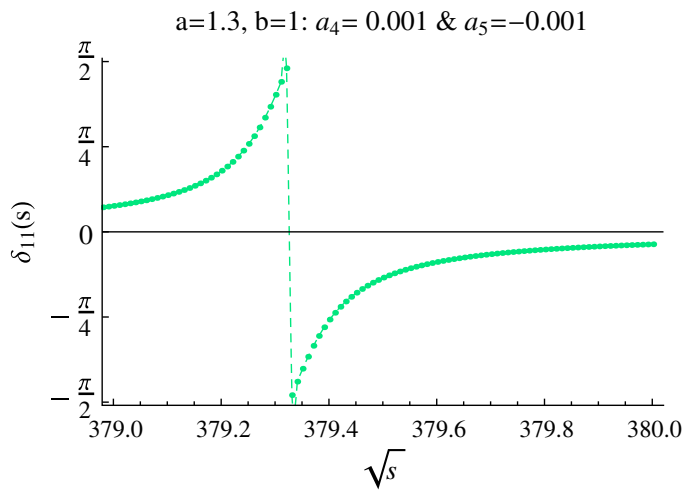

(b)

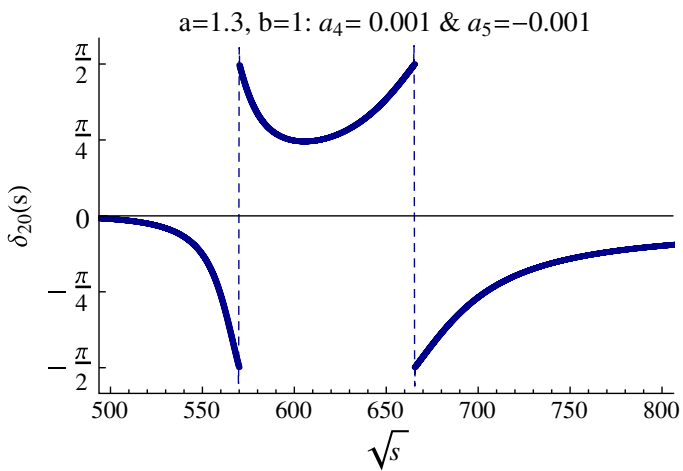

(c)

FIG. 11 (color online). Phase shifts for $a_{4}=0.001$ and $a_{5}=-0.001$ and the values $a=1.3$ and $b=1$. The plots show wrong resonances for the isoscalar $(\simeq 760 \mathrm{GeV})$ and tensor $(\simeq 570 \mathrm{GeV})$ since the shift is from $-\pi / 2$, whereas the isovector has a good resonances $(\simeq 380 \mathrm{GeV})$. Moreover, the second tensor resonances $(\simeq 665 \mathrm{GeV})$ with positive width are also shown. 
for $a=0.95$, which is very close to the MSM value for $a, a=1$.

We have also considered the case where $b=a^{2}$. In this case in the ET approximation, the two channels decouple, and our implementation of the IAM becomes better justified. Results for the cases $a=0.9$ and $b=a^{2}$ are depicted in Fig. 8. Changes with respect to $b=1$ are unnoticeable, indicating that $b$ is of little relevance for the presence of resonances.

Figure 9 shows the masses and widths of the scalar and vector resonances obtained for $a=0.9$. As we see, in general they tend to be heavier and broader than the ones in the $a=1$ case studied in Ref. [10]. We emphasize that the resonance in the scalar channel is additional to the Higgs at $125 \mathrm{GeV}$. The impact of parameter $b$ is actually more visible in the widths of the different resonances. In Fig. 10 we depict the widths obtained in the scalar and vector channels for $b=a^{2}$ when $a=0.9$

\section{B. Case $a>1$}

As we have seen, the $a<1$ case is really a smooth continuation of the $a=1$ limit. Resonances are somewhat more rare, and they tend to be slightly heavier and broader, the more so as one departs from $a=1$, but the modifications are small. This changes when we go to the $a>1$ case.

In this case the tree-level amplitudes $t_{I J}^{(0)}$ tend to $-\infty$ as $s \rightarrow \infty$. In the isoscalar channel for $1<a<1.125$, they possess several additional zeroes, which disappear for $a>1.125$. In the isovector channels, the additional zeroes remain for even larger values of $a$. Past these zeroes, the tree-level contribution is negative all the way up to the limit of validity of the effective theory.

One finds zeroes of the denominator in Eq. (17) that would correspond to resonances provided that the numerator does not vanish. This comment is relevant because many of the resonances present, particularly in the vector channel, appear in the region near the last (as $s$ increases) zero of the amplitude, and this requires particular care. In fact for a set of values of $a_{4}$ and $a_{5}$, the determination as to whether a pole exists or not becomes ambiguous.

When we continue our amplitudes into their second Riemann sheet to estimate the width and solve for the complex pole, we find that in various channels the imaginary part is such that it corresponds to a negative width. When two poles in a given channel are found, one is acceptable, but then the other one leads to acausal behavior (this can be proven analytically). For other values of the coupling, the resonances are perfectly acceptable.

As an example of the pathologies found, we show for $a=1.3$ in Fig. 11 the phase shifts for the isotensor-scalar and isovector channels. We can see a behavior that is incompatible with causality for the isotensor-scalar phase shift; recall that $\Gamma=2\left(\frac{d \delta}{d \sqrt{s}}\right)^{-1}$. Sometimes a bona fide resonance pole coexists with a second resonance having negative width. This can be seen, for instance, in Fig. 12 in the scalar channel for $a=1.1$. We see that one genuine looking resonance coexists with a huge singularity having a large negative width. The corresponding effective theory is unacceptable.

The net result is that a very sizeable part of the space of parameters is ruled out. For instance, in Fig. 13 we show the excluded areas for $a=1.1$, Fig. 13(a), and $a=1.3$, Fig. 13(b). We are thus forced to conclude that pathologies abound in the $a>1$ case. In particular, we have been unable to find a bona fide $I=2$ dynamical resonance for $a=1.1$ and $a=1.3$, and this seems to be the generic situation for $a>1$.

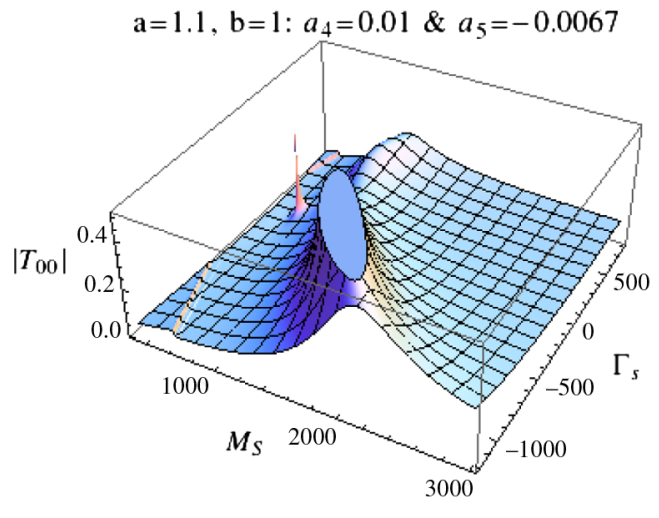

(a)

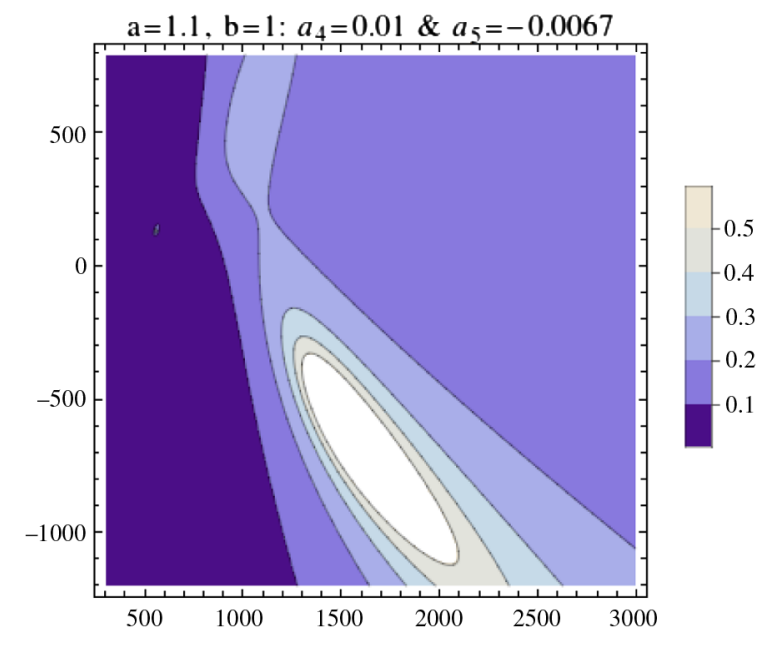

(b)

FIG. 12 (color online). (a) The two resonances that appear in the scalar channel are shown in a three-dimensional plot. The larger one has a large negative width. The corresponding contour plot is shown in (b), where the physical one is nearly invisible, being extremely narrow. 


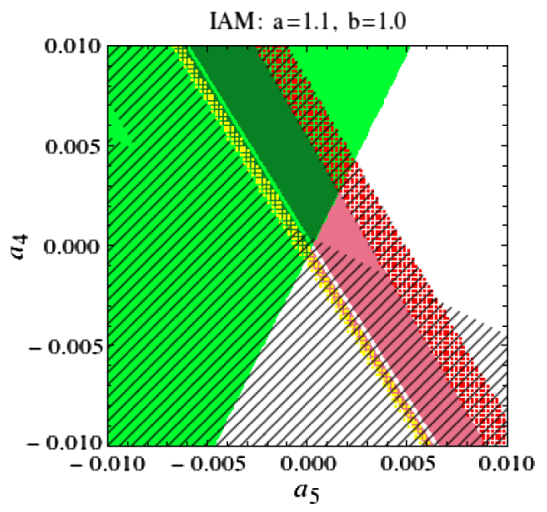

(a)

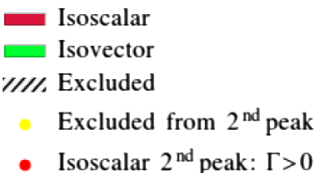

(a) Search for resonances for $a=1.1$ up to the scale $4 \pi v \approx 3 \mathrm{TeV}$. The lower part of parameter space is channel. Some isovector and isoscalar resonances are possible. In the white area in the upper left corner, the scalar resonances are very broad and are not considered as such by the $\Gamma<M / 4$ condition. (b) Same as (a) for $a=1.3$. The areas excluded due to resonances developing negative widths are now even larger. No resonance satisfying our criteria exists in the scalar channel; the apparent poles have all negative widths for $I=0$. In a sizeable area, vector resonances develop a second unphysical resonance. As for the isotensor channel, most of the parameter space has one pole with negative width. Then a second exclusion band (similar to the isovector one for $a=1.1$ ) exists due to isotensor channels having one valid resonance together with a second acausal one. Only a small set of values presents one valid resonance in the isovector channel. Note that $a_{4}=a_{5}=0$ is unphysical for $a=1.3$.

On the other hand, even though our findings basically exclude $I=2$ dynamical resonances for $a>1$, a light and elementary $I=2$ state can be included in the Lagrangian, such as in the Georgi-Machacek model with a light quintuplet [27]. This is not contradictory to our findings. If one wants to consider a weakly coupled state with a mass much below the natural cutoff of the theory, it has to be made explicit in the effective Lagrangian as a propagating degree of freedom. Then it will appear as a pole also after unitarization, exactly as the light Higgs does. In addition there may or may not be dynamical resonances depending on the nature of the short distance theory. The IAM, which is known to work well for strongly coupled theories, seems to be robust enough to support a strongly interacting sector and a perturbative one coexisting in the same theory.

Interestingly, the difference between the $a>1$ and $a<$ 1 regimes looks consistent with the sum rule introduced in Ref. [26]. However, in the Appendix we comment about a possible issue which could affect the derivation of this sum rule.

\section{EXPERIMENTAL VISIBILITY OF THE RESONANCES}

One thing is having a resonance, and a very different one is being able to detect it. In particular, the statistics so far available from the LHC experiments is limited. Searching for new particles in the LHC environment is extremely challenging. Yet a particle with the properties of the Higgs has been found with only limited statistics. This has been possible in part because of a fortunate upward statistical fluctuation but also because the couplings and other properties of the Higgs were well known in the MSM. This is not necessarily the case for new resonances that may exist in the EWSBS. Fortunately the IAM method is able to predict not only masses and widths but also their couplings to the $W_{L} W_{L}$ and $Z_{L} Z_{L}$ channels. In Ref. [10], where the case $a=1$ was considered, the experimental signal of the different resonances was compared to that of a MSM Higgs with an identical mass. Because the decay modes are similar (in the vector boson channels that is) and limits on different Higgs masses are well studied, this is a practical way of presenting the results.

Therefore, to gain some intuition as to whether any of the predicted resonances for $a<1$ should have been seen by now at the LHC, we compare their signal (the size of the corresponding Breit-Wigner resonance) with the one of the Higgs at an equivalent mass. Just to gain some intuition on this, we have used the easy-to-implement effective W approximation (EWA) [28]. The results are depicted in Fig. 14 for the $W_{L} W_{L}$ and $Z_{L} Z_{L}$ vector fusion channels. Note that both production modes are subdominant at the LHC with respect to gluon production mediated by a topquark loop, and also note that the decay modes of the resonances can be predicted with the technology presented here only for $W_{L} W_{L}$ and $Z_{L} Z_{L}$ final states.

What can be seen in these figures is that the signal is always lower than the one for a Higgs boson of an equivalent mass. However, the ratio $\sigma_{\text {resonance }} / \sigma_{\text {Higgs }}$ seems to depend substantially on the value of $a$. For instance, for $a=1$ it was found that in the scalar channel this ratio was typically lower than 0.1 and only in some very limited sector of parameter space could be as large as 0.3. It was even lower for $Z Z$ production. Now for $a=0.9$ we see that 


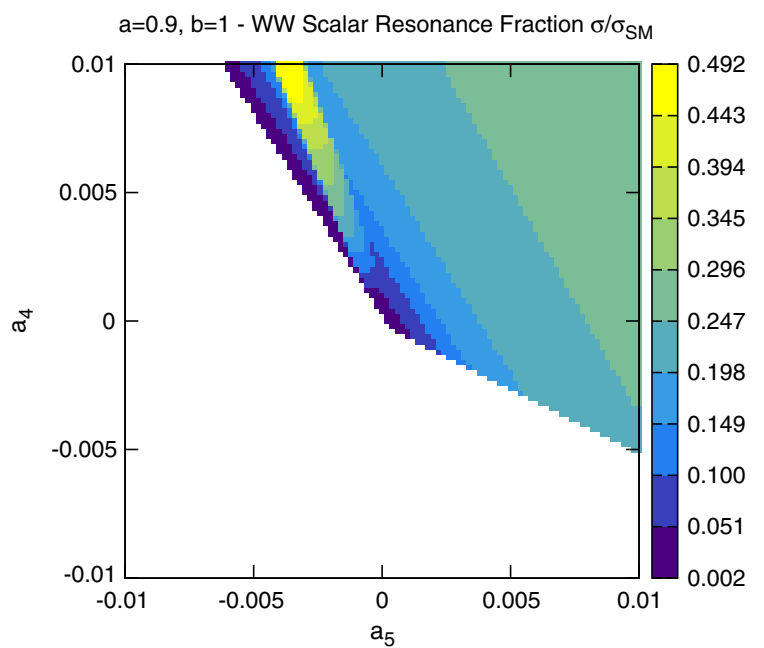

(a)

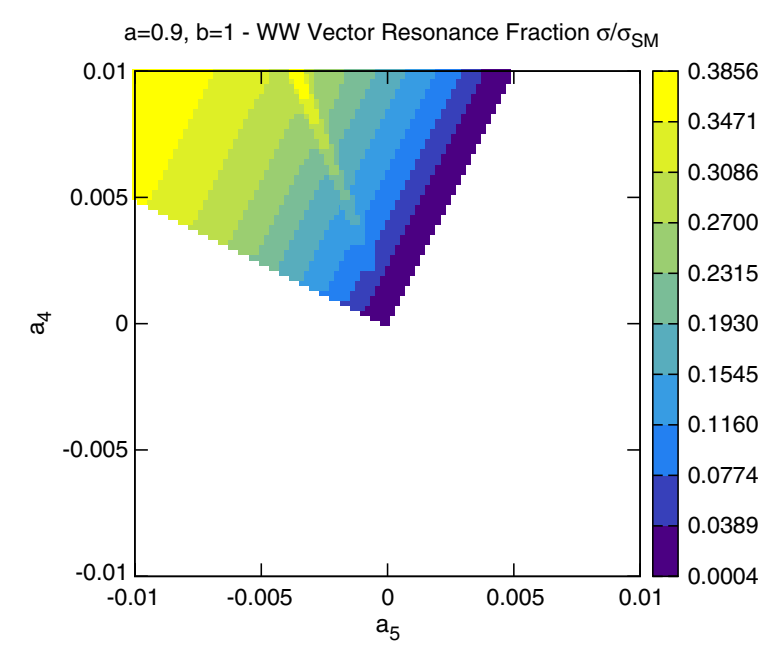

(b)

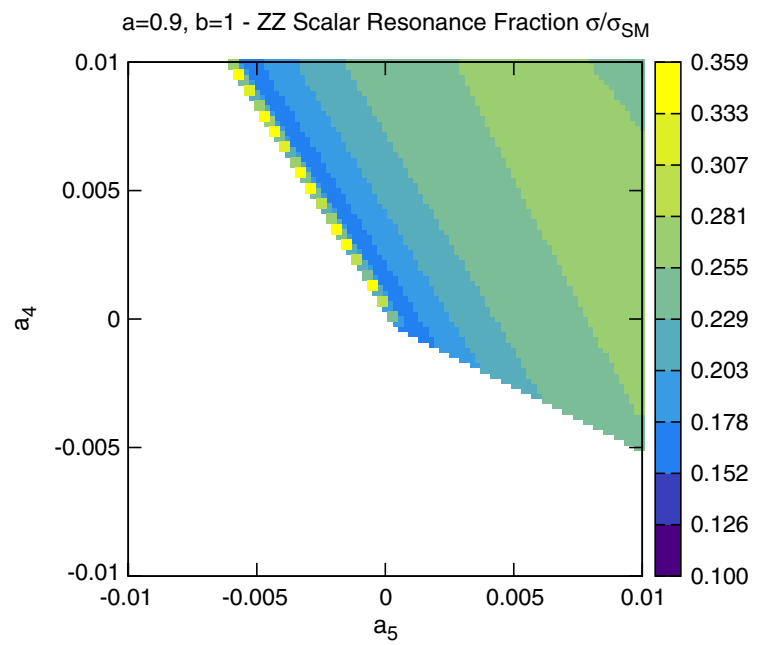

(c)

FIG. 14 (color online). For $a=0.9$ and $b=1$ : Ratios of the $W_{L} W_{L}$ scattering cross section due to dynamical resonances with that of the SM with a Higgs boson of the same mass for (a) scalar and (b) vector resonances, taken in the peak region as defined in Ref. [10]. (c) Ratio of the $Z_{L} Z_{L}$ scattering cross section due to dynamical resonances with that of the SM with a Higgs boson of the same mass for a scalar resonance. The LHC energy has been taken to be $8 \mathrm{TeV}$, and the EWA approximation is assumed.

0.2 is a more typical value for $\sigma_{\text {resonance }} / \sigma_{\text {Higgs }}$ and in some areas of parameter space can go up to $\sim 0.4$ or even close to 0.5 . Again the signal is somewhat lower in the $Z_{L} Z_{L}$ production channel. For the vector channel and again normalizing to the Higgs signal, we get ratios for $\sigma_{\text {resonance }} / \sigma_{\text {Higgs }}$, the signal ranges from 0.03 to 0.3 .

\section{CONCLUSIONS}

In this paper we have extended the analysis of Ref. [10] to the cases $a \neq 1$ and $b \neq 1$, imposing the requirement of unitarity on the fixed isospin amplitudes contributing to longitudinal $W$ scattering. The method chosen to unitarize the partial waves is the inverse amplitude method. The simplicity of this method makes it suitable to analyze the problem being considered, while its validity has been well tested in strong interactions in the past.
We have seen that, even in the presence of a light Higgs, it can help constrain anomalous couplings by helping predict heavier resonances, present in an extended EWSBS. The results for $a \neq 1$ presented here turn out to be partly in line with the results for $a=1$ previously obtained if $a<1$ and partly qualitatively different if $a>1$. If $a<1$ for a large subset of values of the higher-dimensional coefficients, resonances are present. Typically they tend to be heavier and broader than in the $a=1$ case, but only moderately so. They are never like the broad resonances that were entertained in the past in Higgsless models, and this is undoubtedly a consequence of the unitarization that the presence of the Higgs brings about. There is a smaller room for new states once unitarity is required. The properties of the resonance are therefore radically different from the initial expectations concerning $W_{L} W_{L}$ scattering. 
Current LHC Higgs search results do not yet probe the IAM resonances, but it may be possible in the near future. This is particularly true if $a$ departs from its Standard Model value $a=1$. In this case, the resonances become higher and broader with values of $\sigma_{\text {resonance }} / \sigma_{\text {Higgs }}$ close to 0.5 . In any case it seems that LHC@ $14 \mathrm{TeV}$ will be able to probe a reasonable part of the possible parameter space for resonances.

If resonances are found with the properties predicted here, this discovery would immediately indicate that there is an extended EWSBS and that this is likely described by some strongly interacting theory, giving credit to the hypothesis of the Higgs being a composite state-most likely a pseudo-Goldstone boson. It would also provide immediate information on the value of some of the higher-dimensional coefficients in the effective theory, probably much earlier than direct $W_{L} W_{L}$ scattering would allow for a determination of the quartic gauge boson coupling.

We have also found another interesting result, namely, that in the present framework theories with $a>1$ are nearly excluded as the IAM predicts that they lead to resonances that violate causality in a large part of parameter space, the more so as one departs more from $a=1$.

\section{ACKNOWLEDGMENTS}

We thank A. Dobado, J. González-Fraile, M. J. Herrero, J.R. Peláez, and B. Yencho for discussions concerning different aspects of unitarization and effective Lagrangians. We acknowledge the financial support from Project No. FPA2010-20807, Programa Nacional de Proyectos de Investigación Fundamental-Física de Partículas (FPA). Ministerio de Ciencia e Innovación Project No. 2009SGR502, CUR Generalitat de Catalunya, and CPAN (Consolider CSD2007-00042).

\section{APPENDIX A: TREE-LEVEL $W_{L} W_{L}$ SCATTERING AMPLITUDES}

In the isospin limit, $M=M_{Z}=M_{W}$, and with massive $W$, the tree-level and $a_{4,5}$-dependent amplitude for $W_{L}^{+} W_{L}^{-} \rightarrow Z_{L} Z_{L}$ scattering is given by

$$
\begin{aligned}
A_{W^{+} W^{-} \rightarrow Z Z}^{\text {tre } a_{i}}\left(p_{1}, p_{2}, p_{3}, p_{4}\right)= & -2 g^{2}\left(1-g^{2} a_{5}\right)\left(\epsilon_{1} \cdot \epsilon_{2}\right)\left(\epsilon_{3} \cdot \epsilon_{4}\right) \\
& +g^{2}\left(1+g^{2} a_{4}\right)\left[\left(\epsilon_{1} \cdot \epsilon_{4}\right)\left(\epsilon_{2} \cdot \epsilon_{3}\right)+\left(\epsilon_{1} \cdot \epsilon_{3}\right)\left(\epsilon_{2} \cdot \epsilon_{4}\right)\right] \\
& +g^{2}\left\{\left(\frac{1}{\left(p_{1}-p_{3}\right)^{2}-M^{2}}\right)[\right. \\
& -4\left(\left(\epsilon_{1} \cdot \epsilon_{2}\right)\left(p_{1} \cdot \epsilon_{3}\right)\left(p_{2} \cdot \epsilon_{4}\right)+\left(\epsilon_{1} \cdot \epsilon_{4}\right)\left(p_{1} \cdot \epsilon_{3}\right)\left(p_{4} \cdot \epsilon_{2}\right)\right. \\
& \left.+\left(\epsilon_{2} \cdot \epsilon_{3}\right)\left(p_{3} \cdot \epsilon_{1}\right)\left(p_{2} \cdot \epsilon_{4}\right)+\left(\epsilon_{3} \cdot \epsilon_{4}\right)\left(p_{3} \cdot \epsilon_{1}\right)\left(p_{4} \cdot \epsilon_{2}\right)\right) \\
& +2\left(\left(\epsilon_{2} \cdot \epsilon_{4}\right)\left(\left(p_{1} \cdot \epsilon_{3}\right)\left(p_{2}+p_{4}\right) \cdot \epsilon_{1}+\left(p_{3} \cdot \epsilon_{1}\right)\left(p_{2}+p_{4}\right) \cdot \epsilon_{3}\right)\right. \\
& \left.+\left(\epsilon_{1} \cdot \epsilon_{3}\right)\left(\left(p_{2} \cdot \epsilon_{4}\right)\left(p_{1}+p_{3}\right) \cdot \epsilon_{2}+\left(p_{4} \cdot \epsilon_{2}\right)\left(p_{1}+p_{3}\right) \cdot \epsilon_{4}\right)\right) \\
& \left.-\left(\epsilon_{1} \cdot \epsilon_{3}\right)\left(\epsilon_{2} \cdot \epsilon_{4}\right)\left(\left(p_{1}+p_{3}\right) \cdot p_{2}+\left(p_{2}+p_{4}\right) \cdot p_{1}\right)\right] \\
& \left.+\left(p_{3} \leftrightarrow p_{4}\right)\right\}-a^{2} g^{2} M^{2}\left(\frac{\left(\epsilon_{1} \cdot \epsilon_{2}\right)\left(\epsilon_{3} \cdot \epsilon_{4}\right)}{\left(p_{1}+p_{2}\right)^{2}-M_{H}^{2}}\right),
\end{aligned}
$$

where $\epsilon_{i}=\epsilon_{L}\left(p_{i}\right)$. The analogous expression in the ET approximation is much simpler:

$$
A_{w^{+} w^{-} \rightarrow z z}^{\mathrm{tree}+a_{i}}(s)=-\left(\frac{s}{v^{2}}\right)\left(\frac{\left(a^{2}-1\right) s+M_{H}^{2}}{s-M_{H}^{2}}-2\left(\frac{s}{v^{2}}\right)\left(a_{4}\left(1+\cos ^{2} \theta\right)+4 a_{5}\right)\right) .
$$

For completeness, we also give the amplitude for the $W_{L}^{+} W_{L}^{-} \rightarrow h h$ scattering:

$$
\begin{aligned}
A_{W^{+} W^{-} \rightarrow h h}^{\mathrm{tree}}\left(p_{1}, p_{1}, q_{3}, q_{4}\right)= & g^{2}\left(\frac{b}{2}\left(\epsilon_{1} \cdot \epsilon_{2}\right)-\frac{3 a M_{H}^{2}}{2\left(M_{H}^{2}-\left(p_{1}+p_{2}\right)^{2}\right)}\left(\epsilon_{1} \cdot \epsilon_{2}\right)\right. \\
& \left.+a^{2} \frac{g^{2} v^{2}}{4 M^{2}}\left(\frac{\left(\epsilon_{1} \cdot\left(q_{3}-p_{1}\right)\right)\left(\epsilon_{2} \cdot\left(q_{3}-p_{1}\right)\right)-M^{2}\left(\epsilon_{1} \cdot \epsilon_{2}\right)}{M^{2}-\left(q_{3}-p_{1}\right)^{2}}+\left(q_{3} \leftrightarrow q_{4}\right)\right)\right) .
\end{aligned}
$$

In the c.m. reference frame, the expression for $A_{W^{+} W^{-} \rightarrow Z Z}^{\text {tree }+a_{i}}(s, t, u)$ becomes 


$$
\begin{aligned}
A_{W^{+} W^{-} \rightarrow Z Z}^{\mathrm{tree}+a_{i}}(s, t, u)= & \frac{a^{2}\left(s-2 M^{2}\right)^{2}}{v^{2}\left(M_{H}^{2}-s\right)} \\
& +\frac{768 M^{10}-128 M^{8}(5 s+4 t)+32 M^{6}\left(7 s^{2}+8 s t+4 t^{2}\right)}{v^{2}\left(s-4 M^{2}\right)^{2}\left(M^{2}-t\right)\left(-3 M^{2}+s+t\right)} \\
& -\frac{8 M^{4} s\left(5 s^{2}+11 s t+4 t^{2}\right)-M^{2} s^{2}\left(3 s^{2}+18 s t+14 t^{2}\right)+s^{3} t(s+t)}{v^{2}\left(s-4 M^{2}\right)^{2}\left(M^{2}-t\right)\left(-3 M^{2}+s+t\right)} \\
& +\frac{\left.8 a_{5}\left(s-2 M^{2}\right)^{2}+2 a_{4}\left(16 M^{4}-8 M^{2} s+\left(1+\cos ^{2} \theta\right) s^{2}\right)\right)}{v^{4}} .
\end{aligned}
$$

Recall that $A_{W^{+} W^{-} \rightarrow Z Z}^{\text {tree }}(s, t, u)$ for the scattering of longitudinally polarized $W$ is not Lorentz invariant. The expression above is valid in the c.m. frame only.

\section{APPENDIX B: ISSUE OF CROSSING SYMMETRY}

We would like to clarify the issue of crossing symmetry of amplitudes with external $W_{L}$ 's. To this end let us consider just the tree-level contribution in the MSM to the processes $W_{L}^{+} W_{L}^{-} \rightarrow W_{L}^{+} W_{L}^{-}$and $W_{L}^{+} W_{L}^{+} \rightarrow W_{L}^{+} W_{L}^{+}$, respectively.

To keep the formulas simple while making the point, let us consider the limit $s \rightarrow \infty,-t \rightarrow \infty$ in the first process, which is consistent except for $\cos \theta \simeq 1$, and expand in $M^{2} / s$ and $M^{2} / t$. We borrow the results from Ref. [18]. The resulting amplitude is

$$
-g^{2}\left(\frac{M_{H}^{2}}{4 M^{2}}\left[\frac{t}{t-M_{H}^{2}}+\frac{s}{s-M_{H}^{2}}\right]+\frac{s^{2}+t^{2}+s t}{2 s t}-\frac{M_{H}^{2}}{s} \frac{2 M_{H}^{2} t-s(s+t)}{\left(M_{H}^{2}-s\right)\left(M_{H}^{2}-t\right)}\right)+\cdots
$$

In the second process we expand in powers of $M^{2} / u$ and $M^{2} / t$. One then gets

$$
-g^{2}\left(\frac{M_{H}^{2}}{4 M^{2}}\left[\frac{t}{t-M_{H}^{2}}+\frac{u}{u-M_{H}^{2}}\right]+\frac{u^{2}+t^{2}+u t}{2 u t}+\frac{M_{H}^{2}}{t+u\left(M_{H}^{2}-u\right)\left(M_{H}^{2}-t\right)}\right)+\cdots .
$$

The two processes are related by crossing, and one would naively think that the two amplitudes can be related by simply exchanging $s$ and $u$. While this is correct for the first two terms in both equations, it fails for the third. If the reader is worried about the approximations made, more lengthy complete results are given in Ref. [18], and they show the same features.

The reason is that, while crossing certainly holds when exchanging the external 4-vectors, the reference frame in which the two above amplitudes are expressed is different. In both cases they correspond to center-of-mass amplitudes, but after the exchange of momenta, the two systems are boosted, one with respect to the other. Writing the amplitudes in terms of $s, t, u$ gives the false impression that these expressions hold in any reference system, but this is not correct because the polarization vectors are no true 4-vectors.

On the contrary, the amplitudes computed via the ET are manifestly crossing symmetric because they amount to replacing $\epsilon_{L}^{\mu} \rightarrow k^{\mu}$, which is obviously a covariant 4-vector. We insist once more that crossing does hold in any case but is not manifest for the scattering of longitudinal $W$ bosons at the level of Mandelstam variables.

\section{APPENDIX C: ORIGIN OF THE LOGARITHMIC POLES}

Here, we discuss the origins of the three singularities at $s_{0}=M_{H}^{2}, s_{1}=4 M^{2}-M_{H}^{2}$, and $s_{2}=3 M^{2}$ ) entering the $t_{I J}(s)$ amplitudes. These singularities can be tracked back from the terms $1 /\left(s-M_{H}^{2}\right), 1 /\left(t-M^{2}\right)$, and $1 /\left(u-M^{2}\right)$ in the $W_{L}^{+} W_{L}^{-} \rightarrow Z_{L} Z_{L}$ amplitude in Eq. (A4). The origin of the pole at $s_{0}$ is fairly obvious and needs no justification.

As for the other two singularities, the term $1 /\left(t-M^{2}\right)=$ $1 /\left((-1+\cos \theta)\left(-4 M^{2}+s\right) / 2-M^{2}\right)$ has a pole at $s_{3}$ for $\cos \theta=-1$, which under integration in $\cos \theta$ to derive the partial wave amplitude $t_{I J}(s)$ becomes a logarithmic pole as well as for $1 /\left(u-M^{2}\right)=1 /\left((1+\cos \theta)\left(-4 M^{2}+s\right) / 2-\right.$ $\left.M^{2}\right)$ at $\cos \theta=-1$. This explains the presence of the $s_{2}$ pole for $t_{I J}(s)$ amplitudes.

The origin of the pole at $s_{1}$ for $t_{I J}(s)$ amplitudes is more complicated to see. First of all, let us notice that the fixed-isospin amplitudes $T_{I}$ in Eq. (7) are combinations of the $A^{++00}=A\left(W_{L}^{+} W_{L}^{-} \rightarrow Z_{L} Z_{L}\right)$ in Eq. (A4) and its crossed amplitude $A^{++++}=A\left(W_{L}^{+} W_{L}^{+} \rightarrow W_{L}^{+} W_{L}^{+}\right)$. At this point, the term $1 /\left(s-M_{H}^{2}\right)$ in $A^{++00}$, Eq. (A4), transforms for the crossed amplitude $A^{++++}$into $1 /\left(t-M_{H}^{2}\right)=\left(1 /(-1+\cos \theta)\left(-4 M^{2}+s\right) / 2-M_{H}^{2}\right)$. 
Then, for $\cos \theta=-1$ we have a pole at $s_{1}$, and under integration on $\cos \theta$, the amplitude $t_{I J}(s)$ gets a logarithmic pole at $s_{1}$.

Note that these singularities are all below threshold. Note, too, that except for $s_{0}$ they are absent in the ET treatment. For the LHC they appear at values of $s$ corresponding to the replacement $4 M^{2} \rightarrow \sum_{i} q_{i}^{2}$ as the external $W$ are typically off shell.

\section{APPENDIX D: SUM RULE}

In Ref. [26] the sum rule was derived,

$$
\begin{aligned}
\frac{1-a^{2}}{v^{2}}= & \frac{1}{6 \pi} \int_{0}^{\infty} \frac{d s}{s}\left(2 \sigma_{I=0}(s)^{\mathrm{tot}}+3 \sigma_{I=1}(s)^{\mathrm{tot}}\right. \\
& \left.-5 \sigma_{I=2}(s)^{\mathrm{tot}}\right)+c_{\infty},
\end{aligned}
$$

where $\sigma_{I}^{\text {tot }}$ is the total cross section in the isospin channel $I$ and $c_{\infty}$ is the contribution of the $|s| \rightarrow \infty$ contour to the dispersive integral. This latter contribution can sometimes be neglected. This is the case, for instance, in $\pi \pi$ scattering. In the forward direction, it is expected to show a Regge behavior compatible with the neglection of the external part of the circuit.

The interesting result (D1) was derived making full use of the equivalence theorem and setting $M=0$. As we have seen, at low $s$ there are some relevant deviations with respect to the ET predictions when using the proper longitudinal vector boson amplitudes and including the $t$-channel $W$ exchange, and they affect the analytic properties of the amplitude. Let us see how this sum rule is affected by these deviations.

The technique used in Ref. [26] to derive the previous result was to define the function $F(s, t, u)=$ $A_{W^{+} W^{-} \rightarrow Z Z}^{\text {tree }}(s, t, u) / s^{2}$; consider the case $t=0$, corresponding to the forward amplitude, and compute the integral

$$
\oint d s F(s, t, u)
$$

using two different circuits: one around the origin and another one along the cuts in the real axis and closing at infinity (this last contribution actually drops if the amplitudes are assumed to grow slower than $s$ ).

Applying the strict ET, each order in perturbation theory contributes to a given order in an expansion in powers of $s, t, u$. Therefore, if the integral is done in a small circle around the origin, only the tree-level amplitude, Eq. (A2), contributes, and taking both contributions into account results in the result on the left-hand side of Eq. (D1). On the other hand, the integral along the left cut can be related using crossing symmetry to the one on the right cut and eventually leads to the right-hand side of Eq. (D1).

Formulas (A1) and (A2) show clearly that the analytic structure of the full result and the ET one are quite different at low values of $s$. In the exact case and for the tree-level amplitude, we have four poles for $F(s, t, u)$. We assume that $s$ and $t$ are independent variables, and to make this visible we replace $t \rightarrow \bar{t}$ :

$$
\begin{gathered}
s_{0}=0 \rightarrow \operatorname{Res}\left(F\left(s_{0}, \bar{t}, u\right)\right)=\frac{4 a^{2} M^{2}\left(M^{2}-M_{H}^{2}\right)}{M_{H}^{4} v^{2}}+\frac{2 \bar{t}\left(8 M^{4}-7 M^{2} \bar{t}+\bar{t}^{2}\right)}{v^{2}\left(M^{2}-\bar{t}\right)\left(\bar{t}-3 M^{2}\right)^{2}} \\
s_{1}=M_{H}^{2} \rightarrow \operatorname{Res}\left(F\left(s_{1}, \bar{t}, u\right)\right)=-\frac{a^{2}\left(M_{H}^{2}-2 M^{2}\right)^{2}}{M_{H}^{4} v^{2}} \\
s_{2}=3 M^{2}-\bar{t} \rightarrow \operatorname{Res}\left(F\left(s_{2}, \bar{t}, u\right)\right)=-\frac{-27 M^{8}+52 M^{6} \bar{t}+M^{4} \bar{t}^{2}+2 M^{2} \bar{t}^{3}}{v^{2}\left(\bar{t}-3 M^{2}\right)^{2}\left(M^{2}+\bar{t}\right)^{2}} \\
s_{3}=4 M^{2} \rightarrow \operatorname{Res}\left(F\left(s_{3}, \bar{t}, u\right)\right)=\bar{t} \frac{10 M^{4}-3 M^{2} \bar{t}-3 \bar{t}^{2}}{\left(M^{2}-\bar{t}\right)\left(M^{2}+\bar{t}\right)^{2} v^{2}} \\
\sum_{i=0,3} \operatorname{Res}\left(F\left(s_{i}, \bar{t}, u\right)\right)=\frac{\left(3-a^{2}\right) M^{2}-\left(1-a^{2}\right) \bar{t}}{\left(M^{2}-\bar{t}\right) v^{2}} .
\end{gathered}
$$

Note, however, that for $s=s_{3}=4 M^{2}$ the $t$ variable is always zero, being $t=-(1-\cos \theta)\left(s-4 M^{2}\right) / 2$, and $u=-(1+\cos \theta)\left(s-4 M^{2}\right) / 2$. This shows that $s$ and $t$ are dependent for some exceptional kinematical points, for example, when the initial states are at rest $\left(s=s_{3}=4 M^{2}\right)$. Therefore, when $s \rightarrow s_{3}, t \rightarrow 0$. If we set $\bar{t}=0$ at the outset, the sum of residues leads to

$$
\sum_{i=0,3} \operatorname{Res}\left(F\left(s_{i}, \bar{t}=0, u\right)\right)=\frac{\left(3-a^{2}\right)}{v^{2}}
$$

which differs from the result quoted in Ref. [26]. The reason is clear when looking at Eq. (D4): if we take the limit $M \rightarrow 0$ at the outset as is done in the strict ET approximation, we get one result, while if $\bar{t}$ is set to zero with $M \neq 0$, we get a different one. 
In addition, in a complete calculation (as opposed to the simpler ET treatment), it is not true that a given order in the chiral expansion corresponds to a definite power of $s$. Therefore, when $M$ is not neglected, the order $s$ contribution will have corrections from all orders in perturbation theory. The contribution to the left-hand side of the integral, obtained after circumnavigating all the poles, will then be of the form

$$
\frac{3-a^{2}+\mathcal{O}\left(g^{2}\right)}{v^{2}}
$$

Actually, the right cut changes, too, when $M$ is taken to be nonzero; it starts at $s=4 M^{2}$ (which is not a pole as we have just discussed because it has a vanishing residue). The left cut is not changed as for $t=0$, and the $u$ channel has a cut for $s<0$ corresponding to $u>4 M^{2}$.

Although crossing symmetry is not manifest (see Appendix B) for the full amplitudes, it remains valid ${ }^{6}$ for $t=0$, and it is possible to relate exactly the contribution along the left cut to the analogous integral along the right one.

\footnotetext{
${ }^{6} \mathrm{We}$ thank the referee for pointing this out to us.
}

[1] G. Aad et al. (The ATLAS Collaboration), Phys. Lett. B 716, 1 (2012).

[2] S. Chatrchyan et al. (The CMS Collaboration), Phys. Lett. B 716, 30 (2012).

[3] M. Redi and A. Tesi, J. High Energy Phys. 10 (2012) 166; G. Panico, M. Redi, A. Tesi, and A. Wulzer, J. High Energy Phys. 03 (2013) 051.

[4] D. Buttazzo, G. Degrassi, P. P. Giardino, G. F. Giudice, F. Sala, A. Salvio, and A. Strumia, J. High Energy Phys. 12 (2013) 089; G. Degrassi, S. Di Vita, J. Elias-Miro, J. R. Espinosa, G. F. Giudice, G. Isidori, and A. Strumia, J. High Energy Phys. 08 (2012) 098.

[5] A. Pomarol and F. Riva, J. High Energy Phys. 01 (2014) 151; M. Montull, F. Riva, E. Salvioni, and R. Torre, Phys. Rev. D 88, 095006 (2013); P. P. Giardino, K. Kannike, I. Masina, M. Raidal, and A. Strumia, J. High Energy Phys. 05v (2014) 046; T. Alanne, S. Di Chiara, and K. Tuominen, J. High Energy Phys. 01 (2014) 041; B. Dumont, S. Fichet, and G. von Gersdorff, J. High Energy Phys. 07 (2013) 065.

[6] I. Brivio, T. Corbett, O. J. P. Eboli, M. B. Gavela, J. Gonzalez-Fraile, M. C. Gonzalez-Garcia, L. Merlo, and S. Rigolin, J. High Energy Phys. 03 (2014) 024.

[7] J. Elias-Miro, J. R. Espinosa, E. Masso, and A. Pomarol, J. High Energy Phys. 11 (2013) 066; , 08 (2013) 033; E. E. Jenkins, A. V. Manohar, and M. Trott, J. High Energy Phys. 10 (2013) 087; , 10 (2013) 087; C. Grojean, E. E. Jenkins, A. V. Manohar, and M. Trott, J. High Energy Phys. 04 (2013) 016; G. Buchalla, O. Cat, and C. Krause, Nucl. Phys. B880, 552 (2014).

[8] G. F. Giudice, C. Grojean, A. Pomarol, and R. Rattazzi, J. High Energy Phys. 06 (2007) 045; R. Contino, M. Ghezzi, C. Grojean, M. Muhlleitner, and M. Spira, J. High Energy Phys. 07 (2013) 035; R. Alonso, M. B. Gavela, L. Merlo, S. Rigolin, and J. Yepes, Phys. Lett. B 722, 330 (2013).

[9] A. Dobado, D. Espriu, and M. J. Herrero, Phys. Lett. B 255, 405 (1991); D. Espriu and M. J. Herrero, Nucl. Phys. B373, 117 (1992); M. J. Herrero and E. Ruiz-Morales, Nucl. Phys. B418, 431 (1994); B437, 319 (1995); D. Espriu and J. Matias, Phys. Lett. B 341, 332 (1995); A. Dobado, M. J.
Herrero, J. R. Peláez, and E. Ruiz-Morales, Phys. Rev. D 62, 055011 (2000); R. Foadi, M. Jarvinen, and F. Sannino, Phys. Rev. D 79, 035010 (2009).

[10] D. Espriu and B. Yencho, Phys. Rev. D 87, 055017 (2013).

[11] R. Delgado, A. Dobado, and F. Llanes, J. High Energy Phys. 02 (2014) 121.

[12] A. Falkowski, F. Riva, and A. Urbano, J. High Energy Phys. 11 (2013) 111.

[13] A. Pich, I. Rosell, and J. J. Sanz-Cillero, Phys. Rev. Lett. 110, 181801 (2013); J. High Energy Phys. 01 (2014) 157.

[14] S. Chatrchyan et al. (The CMS Collaboration), Report No. CMS-PAS-SMP-13-005; G. Aad et al. (The ATLAS Collaboration), Report No. ATLAS-CONF-2013-020.

[15] S. Chatrchyan et al. (The CMS Collaboration), J. High Energy Phys. 07 (2013) 116.

[16] D. Espriu, F. Mescia, and B. Yencho, Phys. Rev. D 88, 055002 (2013).

[17] J. M. Cornwall, D. N. Levin, and G. Tiktopoulos, Phys. Rev. D 10, 1145 (1974); C. E. Vayonakis, Lett. Nuovo Cimento Soc. Ital. Fis. 17, 383 (1976).; B. W. Lee, C. Quigg, and H. B. Thacker, Phys. Rev. D 16, 1519 (1977); G. J. Gounaris, R. Kogerler, and H. Neufeld, Phys. Rev. D 34, 3257 (1986); M.S. Chanowitz and M. K. Gaillard, Nucl. Phys. B261, 379 (1985); A. Dobado and J. R. Pelaez, Nucl. Phys. B425, 110 (1994); Phys. Lett. B 329, 469 (1994); C. Grosse-Knetter and I. Kuss, Z. Phys. C 66, 95 (1995); H. J. He, Y. P. Kuang, and X. Li, Phys. Lett. B 329, 278 (1994).

[18] D. Espriu and J. Matias, Phys. Rev. D 52, 6530 (1995).

[19] R. Delgado, A. Dobado, and M. J. Herrero (private communication).

[20] T. N. Truong, Phys. Rev. Lett. 61, 2526 (1988); A. Dobado, M. J. Herrero, and T. N. Truong, Phys. Lett. B 235, 134 (1990); A. Dobado and J. R. Pelaez, Phys. Rev. D 47, 4883 (1993); 56, 3057 (1997); J. A. Oller, E. Oset, and J. R. Pelaez, Phys. Rev. Lett. 80, 3452 (1998); Phys. Rev. D 59, 074001 (1999); 60, 099906 (1999); F. Guerrero and J. A. Oller, Nucl. Phys. B537, 459 (1999); A. Dobado and J. R. Pelaez, Phys. Rev. D 65, 077502 (2002); A. Filipuzzi, J. Portoles, and P. Ruiz-Femenia, J. High Energy Phys. 08 (2012) 080; Proc. Sci., CD12 (2013) 053. 
[21] A. D. Martin and T.D. Spearman, Elementary Particle Theory (North-Holland, Amsterdam, 1970).

[22] A. Denner, S. Dittmaier, and T. Hahn, Phys. Rev. D 56, 117 (1997); A. Denner and T. Hahn, Nucl. Phys. B525, 27 (1998).

[23] R. Delgado, A. Dobado, and F. Llanes, J. Phys. G 41, 025002 (2014).

[24] J. Gasser and H. Leutwyler, Ann. Phys. (Berlin) 158, 142 (1984); Nucl. Phys. B250, 465 (1985); B250, 517 (1985).
[25] D. Espriu and P. Ciafaloni, Phys. Rev. D 56, 6885 (1997).

[26] A. Falkowski, S. Rychkov, and A. Urbano, J. High Energy Phys. 04 (2012) 073; A. Urbano, arXiv:1310.5733.

[27] H. Georgi and M. Machacek, Nucl. Phys. B262, 463 (1985).

[28] G. L. Kane, W. W. Repko, and W. B. Rolnick, Phys. Lett. 148B, 367 (1984); S. Dawson, Phys. B 249, 42 (1985); M. S. Chanowitz and M. K. Gaillard, Nucl. Phys. B261, 379 (1985). 